\title{
THE DOUBLY-TRUNCATED EXPONENTIATED INVERSE WEIBULL DISTRIBUTION
}

\section{Demet AYDIN *}

\author{
Department of Statistics, Faculty of Science and Letters, Sinop University, Sinop, Turkey
}

\begin{abstract}
In this study, we introduce the five-parameter doubly-truncated exponentiated inverse Weibull distribution, where the truncation points are known, and consider its basic properties. Formulas for moment generating function, characteristic function, mean, variance and kurtosis of the distribution are derived. Estimates of parameters are obtained using traditional estimation methods; maximum likelihood, least squares and weighted least squares. Furthermore, in order to give a lower bound for the standard errors of estimates of parameters, a Fisher information matrix is provided. A Monte-Carlo simulation study is designed and conducted to evaluate the performances of obtained estimators of parameters. Finally, a real data example is given to show the applicability of the proposed distribution.
\end{abstract}

Keywords: Doubly-truncated exponentiated inverse Weibull distribution, Maximum likelihood method, Fisher information matrix, Monte-Carlo simulation

\section{INTRODUCTION}

The family of exponentiated distributions, which is a new family of distributions named exponentiated exponential distribution, was introduced by Gupta et al. [10]. In particular, let $X$ denote a random variable, and then the cumulative density function $(c d f)$ of a family of exponentiated distributions is defined by

$$
G_{E}(x)=(F(x))^{\theta} ; \theta>0, x \in \mathbb{R},
$$

where $F(x)$ is the $c d f$ of $X$. Gupta and Kundu [11] studied certain properties of the distribution. They observed that many properties of the new family are quite similar to those of the Weibull or Gamma family. For this reason, they stated that this distribution can be used as an alternative to Weibull or Gamma distribution.

In reliability studies, the inverse Weibull distribution introduced by Keller et al. [15] is commonlyused to model a variety of failure characteristics, such as infant mortality, failures of mechanical components subject to degradation, useful life and wear-out periods; see Calabria and Pulcini [5], Cohen and Whitten [6], de Gusmão et al. [8], Khan et al. [17], Lawless [18], and Jiang et al. [12]. If a two-parameter inverse Weibull distribution is written instead of the $F(x)$ given in (1), the $c d f$ of threeparameter exponentiated inverse Weibull $(E I W)$ distribution is obtained as

$$
G(x)=e^{-\alpha \theta x^{-\beta}} ; \alpha>0, \beta>0, x>0,
$$

where $\alpha$ is the scale parameter and $\beta$ and $\theta$ are the shape parameters. Moreover, in the literature, various different distributions have been considered by a number of authors in the context of exponentiated distributions, for example; exponentiated Weibull, exponentiated Exponential and exponentiated Pareto distributions (Gupta and Kundu [10]); exponentiated Gamma, exponentiated Fréchet and exponentiated Gumbel distributions (Nadarajah and Kotz [20]); and exponentiated inverse Weibull, exponentiated Logistic, exponentiated generalized Uniform, exponentiated general

* Corresponding Author: daydin@sinop.edu.tr 
Exponential, exponentiated double Exponential, exponentiated double Weibull and exponentiated double inverse Weibull (Ali et al. [3]).

The graphs of different density functions of $E I W$ distribution for certain selected values of parameters are given in Figure 1. From Figure 1, it can be seen that the density function strictly increases on $\left(0, X_{m}\right]$ and strictly decreases on $\left[X_{m}, \infty\right)$, where $X_{m}$ is the mode of $E I W$ distribution (see, Marusic et al. [19]), and $E I W$ distribution also has a heavy right tail since $\lim _{x \rightarrow \infty} \sup f(x) e^{k x}=\infty$ for all $k>0$, where $f(x)$ is $p d f$ of $E I W$ distribution (Foss et. al. [9]).

On the other hand, truncated distributions are applicable to cases when the range of a random variable is limited from below and/or above for different reasons, and also this situation is commonlyencountered in life-testing and reliability analyses (Zhang and Xie [25]). Since the doubly truncated case of a distribution includes the lower truncated, upper truncated and non-truncated distributions as special cases, it is the most general case (Al-Matrafi and Jawa [4]). Ahmad [2], Coffey and Muller [7], Joshi [14], Okasha and Alqanoo [21], and Shah and Jaiswal [23] are among those who have investigated doubly truncated distributions. Now, the $c d f$ of a doubly truncated distribution can be written as a general formula

$$
G_{D T}(x)=\frac{F(x)-F\left(t_{0}\right)}{F\left(t_{1}\right)-F\left(t_{0}\right)} ; t_{0} \leq x \leq t_{1}
$$

see Zhang and Xie [25]. Here, $F(x)$ is the $c d f$ of $X, t_{0}$ is the lower truncation point and $t_{1}$ is the upper truncation point. If $F(x)$ is taken as the EIW distribution given in (2), the $c d f$ of the doubly truncated $E I W(D T E I W)$ distribution with a lower truncation point $t_{0}$ and an upper truncation point $t_{1}$ can be obtained as

$$
F(x ; \alpha, \beta, \theta)=\frac{e^{-\alpha \theta x^{-\beta}}-e^{-\alpha \theta t_{0}}{ }^{-\beta}}{e^{-\alpha \theta t_{1}-\beta}-e^{-\alpha \theta t_{0}}{ }^{-\beta}} ; \alpha>0, \beta>0, \theta>0, t_{0}<x<t_{1} .
$$

It should be noted that $F(x ; \alpha, \beta, \theta)$ in (3) contains both the same scale parameter $(\alpha)$ and shape parameters $(\beta$ and $\theta$ ) based on the characteristics of the usual EIW distribution and truncation parameters $\left(t_{0}\right.$ and $\left.t_{1}\right)$. In (3), when $t_{0} \rightarrow 0$ and $t_{1} \rightarrow \infty$, the doubly truncated distribution becomes a usual three-parameter $E I W$ distribution. When $t_{0} \rightarrow 0$, it is the upper truncated $E I W$ distribution and when $t_{1} \rightarrow \infty$, it is the lower truncated $E I W$ distribution. Therefore, the probability density function (pdf) of DTEIW distribution is

$$
f(x ; \alpha, \beta, \theta)=\frac{\alpha \theta \beta x^{-(\beta+1)} e^{-\alpha \theta x^{-\beta}}}{e^{-\alpha \theta t_{1}-\beta}-e^{-\alpha \theta t_{0}-\beta}} ; \alpha>0, \beta>0, \theta>0, t_{0}<x<t_{1} .
$$

Plots of an EIW and a DTEIW distributions with lower and upper truncation points, $t_{0}$ and $t_{1}$, for selected $\alpha, \beta$ and $\theta$ values, are given in Figure 2. In Figure 2, the dotted blue and solid red lines represent $E I W$ and DTEIW distributions, respectively.

Then the corresponding reliability function is

$$
R(x ; \alpha, \beta, \theta)=\frac{e^{-\alpha \theta t_{1}-\beta}-e^{-\alpha \theta x^{-\beta}}}{e^{-\alpha \theta t_{1}-\beta}-e^{-\alpha \theta t_{0}}-\beta}
$$

and the hazard function of DTEIW distribution is

$$
h(x ; \alpha, \beta, \theta)=\frac{\alpha \theta \beta x^{-(\beta+1)} e^{-\alpha \theta x^{-\beta}}}{e^{-\alpha \theta t_{1}^{-\beta}}-e^{-\alpha \theta x^{-\beta}}} .
$$


Plots of different hazard functions of EIW and DTEIW distributions for certain $\alpha, \beta$ and $\theta$ values are given in Figures 3-4, separately.

The aim of this paper is to introduce five-parameter DTEIW distribution where the truncation points are known, to study certain statistical properties of the distribution, to obtain estimators of the parameters of the DTEIW distribution using different estimation methods, and to compare the performances of obtained estimators.

The rest of the paper is arranged as follows. We present DTEIW distribution and consider its statistical properties in Section 2. In Section 3, we explain how to obtain estimates of parameters via different estimation methods for DTEIW distribution. In Section 4, the performances of the considered estimation methods in Section 3 are evaluated using a Monte-Carlo simulation study. In Section 5, we analyse a real data set. The paper ends with conclusions in Section 6.

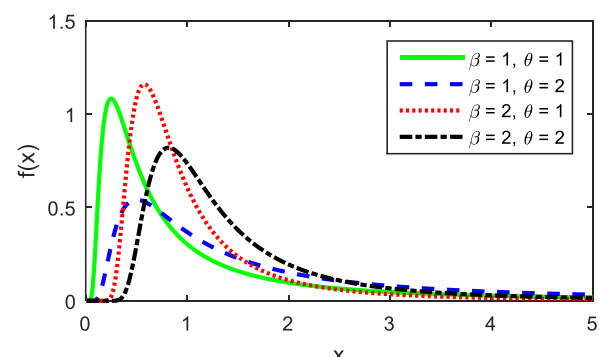

(a)

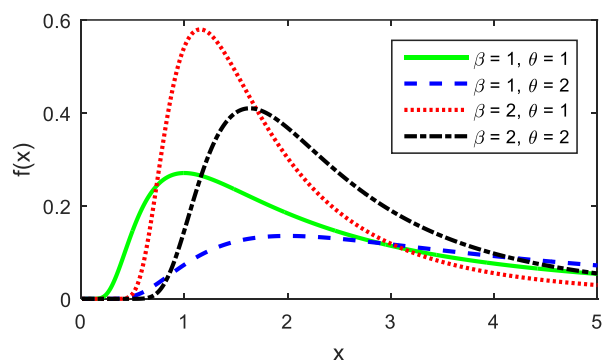

(c)

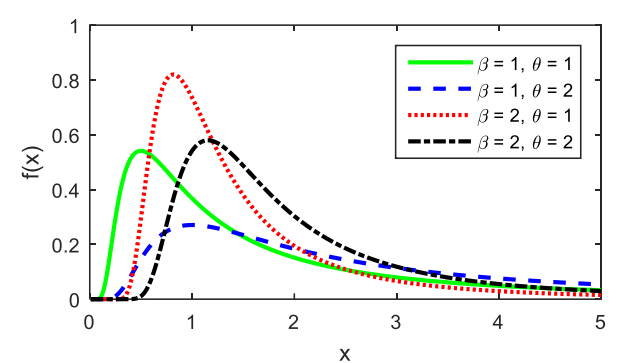

(b)

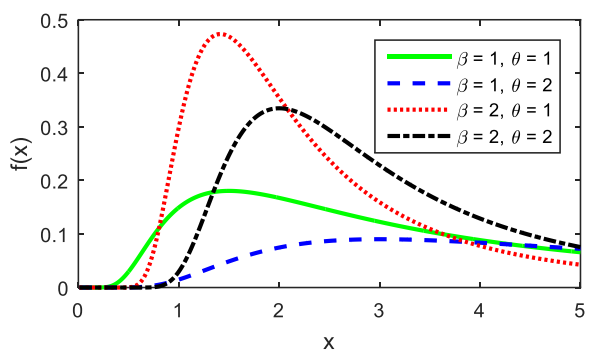

(d)

Figure 1. Plots of different density functions of $E I W$ distribution for $(\beta, \theta)=(1,1),(1,2),(2,1),(2,2)$ and (a) $\alpha=0.5$, (b) $\alpha=$ 1, (c) $\alpha=2$ and (d) $\alpha=3$.

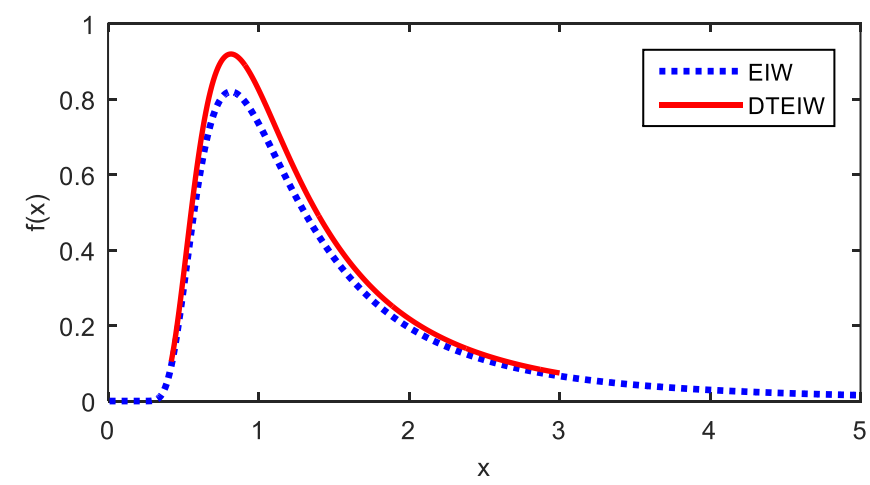

Figure 2. Plots of $p d f \mathrm{~s}$ of $E I W$ (blue line) and DTEIW (red line) distributions superimposed for $\alpha=1, \beta=2$ and $\theta=1$, where the truncation points are taken as $t_{0}=0.42$ and $t_{1}=3$. 
Aydın / Anadolu Univ. J. of Sci. and Technology B - Theo. Sci. 6 (1) - 2018

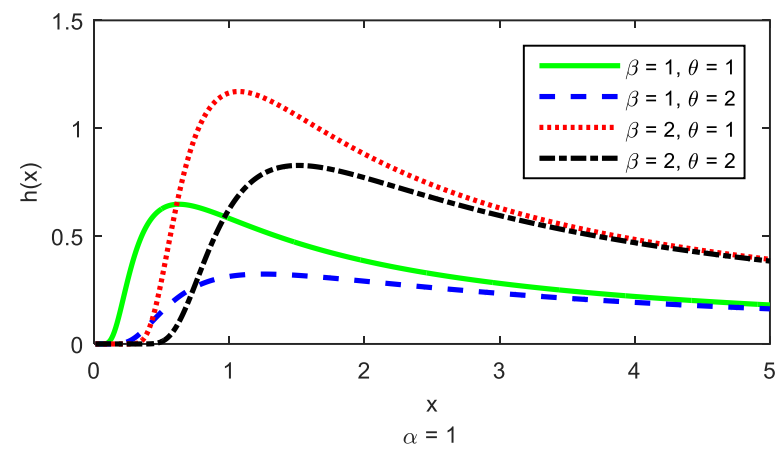

Figure 3. Plots of different hazard functions of EIW distribution for $(\alpha, \beta, \theta)=(1,1,1),(1,1,2),(1,2,1),(1,2,2)$.

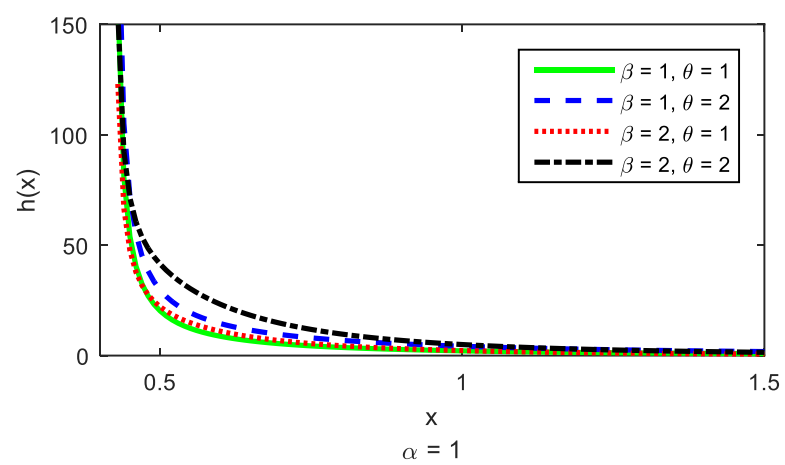

Figure 4. Plots of different hazard functions of DTEIW distribution for $(\alpha, \beta, \theta)=(1,1,1),(1,1,2),(1,2,1),(1,2,2)$, where the truncation points are taken as $t_{0}=0.42$ and $t_{1}=3$.

\section{STATISTICAL PROPERTIES OF DTEIW DISTRIBUTION}

This section discusses how certain statistical properties, such as $k$ th moment, moment generating function, skewness and kurtosis of DTEIW distribution are derived.

Proposition 1: The $k$ th moment of a DTEIW distributed random variable $X$ can be obtained as

$$
E\left(X^{k}\right)=\int_{t_{0}}^{t_{1}} x^{k} \frac{\alpha \theta \beta x^{-(\beta+1)} e^{-\alpha \theta x^{-\beta}}}{e^{-\alpha \theta t_{1}^{-\beta}}-e^{-\alpha \theta t_{0}-\beta}} d x .
$$

In order to calculate $E\left(X^{k}\right)$, we apply the following transformation

$$
u=\alpha \theta x^{-\beta} \Rightarrow x=\left(\frac{u}{\alpha \theta}\right)^{-1 / \beta} \Rightarrow d u=-\alpha \theta \beta x^{-(\beta+1)} d x
$$

and obtain

$$
E\left(X^{k}\right)=\frac{(\alpha \theta)^{k / \beta}}{T} \int_{\alpha \theta t_{1}-\beta}^{\alpha \theta t_{0}^{-\beta}} u^{-k / \beta} e^{-u} d u,
$$

where $T=e^{-\alpha \theta t_{1}{ }^{-\beta}}-e^{-\alpha \theta t_{0}{ }^{-\beta}}$.

Integrating (6), we get 


$$
E\left(X^{k}\right)=\frac{(\alpha \theta)^{k / \beta}}{T}\left(\gamma\left(1-k / \beta, \alpha \theta t_{0}{ }^{-\beta}\right)-\gamma\left(1-k / \beta, \alpha \theta t_{1}{ }^{-\beta}\right)\right),
$$

where $\gamma(.,$.$) denotes the lower incomplete gamma function with \gamma(a, x)=\int_{0}^{x} u^{a-1} e^{-u} d u$.

The distributional characteristics of DTEIW, such as expectation, variance, skewness and kurtosis, can then easily be obtained as a result of Proposition 1.

Corollary 1: The expected value of random variable $X$ is

$$
E(X)=\frac{(\alpha \theta)^{1 / \beta}}{T}\left(\gamma\left(1-1 / \beta, \alpha \theta t_{0}{ }^{-\beta}\right)-\gamma\left(1-1 / \beta, \alpha \theta t_{1}^{-\beta}\right)\right) .
$$

Corollary 2: The variance value of random variable $X$ is

$$
\begin{aligned}
\operatorname{Var}(X)= & \frac{(\alpha \theta)^{2 / \beta}}{T}\left(\gamma\left(1-2 / \beta, \alpha \theta t_{0}{ }^{-\beta}\right)-\gamma\left(1-2 / \beta, \alpha \theta t_{1}{ }^{-\beta}\right)\right) \\
& -\frac{(\alpha \theta)^{2 / \beta}}{T^{2}}\left(\gamma\left(1-1 / \beta, \alpha \theta t_{0}{ }^{-\beta}\right)-\gamma\left(1-1 / \beta, \alpha \theta t_{1}{ }^{-\beta}\right)\right)^{2} .
\end{aligned}
$$

Corollary 3: The skewness of random variable $X$ is

$$
\beta_{1}=\frac{E\left(X^{3}\right)-3 E(X) E\left(X^{2}\right)+2 E(X)^{3}}{\operatorname{Var}(X)^{3 / 2}},
$$

which can be easily calculated by from (7).

Corollary 4: The kurtosis of random variable $X$ is

$$
\beta_{2}=\frac{E\left(X^{4}\right)-4 E(X) E\left(X^{3}\right)+6 E(X)^{2} E\left(X^{2}\right)-4 E(X)^{4}}{\operatorname{Var}(X)^{2}}-3,
$$

which can be obtained using (7).

Proposition 2: The moment generating function of $X$ having DTEIW distribution is

$$
E\left(e^{t x}\right)=\int_{t_{0}}^{t_{1}} e^{t x} \frac{\alpha \theta \beta x^{-(\beta+1)} e^{-\alpha \theta x^{-\beta}}}{e^{-\alpha \theta t_{1}^{-\beta}}-e^{-\alpha \theta t_{0}}-\beta} d x .
$$

Substituting the Maclaurin series for $e^{t x}$ into (8), we obtain the following moment generating function of the DTEIW distributed random variable $X$

$$
\begin{aligned}
E\left(e^{t x}\right) & =\frac{1}{T} \sum_{m=0}^{\infty} \frac{t^{m}}{m !} \int_{t_{0}}^{t_{1}} \alpha \theta \beta x^{m-(\beta+1)} e^{-\alpha \theta x^{-\beta}} d x \\
& =\frac{1}{T} \sum_{m=0}^{\infty} \frac{(\alpha \theta)^{m / \beta} t^{m}}{m !}\left(\gamma\left(1-m / \beta, \alpha \theta t_{0}{ }^{-\beta}\right)-\gamma\left(1-m / \beta, \alpha \theta t_{1}{ }^{-\beta}\right)\right),
\end{aligned}
$$

where $T=e^{-\alpha \theta t_{1}^{-\beta}}-e^{-\alpha \theta t_{0}^{-\beta}}$.

Proposition 3: The characteristic function of $X$ is obtained by

$$
E\left(e^{i t x}\right)=\int_{t_{0}}^{t_{1}} e^{i t x} \frac{\alpha \theta \beta x^{-(\beta+1)} e^{-\alpha \theta x^{-\beta}}}{e^{-\alpha \theta t_{1}-\beta}-e^{-\alpha \theta t_{0}-\beta}} d x .
$$


Using the Maclaurin series for $e^{i t x}$, we have

$$
\begin{aligned}
E\left(e^{i t x}\right) & =\frac{1}{T} \sum_{m=0}^{\infty} \frac{(i t)^{m}}{m !} \int_{t_{0}}^{t_{1}} \alpha \theta \beta x^{m-(\beta+1)} e^{-\alpha \theta x^{-\beta}} d x \\
& =\frac{1}{T} \sum_{m=0}^{\infty} \frac{(\alpha \theta)^{m / \beta}(i t)^{m}}{m !}\left(\gamma\left(1-m / \beta, \alpha \theta t_{0}{ }^{-\beta}\right)-\gamma\left(1-m / \beta, \alpha \theta t_{1}{ }^{-\beta}\right)\right),
\end{aligned}
$$

where $T=e^{-\alpha \theta t_{1}^{-\beta}}-e^{-\alpha \theta t_{0}^{-\beta}}$.

Proposition 4: The quantile function of the random variable $X$ with $p d f$ in (4) is obtained by inverting (3) for $0<q<1$,

$$
x_{q}=\left(-\frac{1}{\alpha \theta} \ln \left(q T+\left(e^{-\alpha t_{0}-\beta}\right)^{\theta}\right)\right)^{-1 / \beta},
$$

where $T=e^{-\alpha \theta t_{1}{ }^{-\beta}}-e^{-\alpha \theta t_{0}{ }^{-\beta}}$.

Corollary 5: The median of a DTEIW distributed random variable $X$ can be found by writing $q=0.5$ in (9). Then we have

$$
\operatorname{Med}(X)=\left(-\frac{1}{\alpha \theta} \ln \left(0.5 T+\left(e^{-\alpha t_{0}-\beta}\right)^{\theta}\right)\right)^{-1 / \beta},
$$

where $T=e^{-\alpha \theta t_{1}{ }^{-\beta}}-e^{-\alpha \theta t_{0}{ }^{-\beta}}$.

Proposition 5: The mode of random variable $X$ having DTEIW distribution is found by differentiating (4) with respect to $x$, equating to zero

$$
\frac{d}{d x} f(x ; \alpha, \beta, \theta)=\frac{1}{T} \alpha \theta \beta x^{-(\beta+2)} e^{-\alpha \theta x^{-\beta}}\left(\alpha \theta \beta x^{-\beta}-(\beta+1)\right)=0,
$$

and then solving for $x$ of (10)

$$
X_{m}=\left(\frac{\alpha \theta \beta}{\beta+1}\right)^{1 / \beta} \text {. }
$$

It should be noted that DTEIW distribution is unimodal because it has a single mode, and the mode of DTEIW distribution in (11) is the same as that of usual EIW distribution; see Fig. 2.

Proposition 6: Suppose that $\left\{X_{1}, X_{2}, \cdots, X_{n}\right\}$ is a random sample of size $n$ from the DTEIW distribution with parameters $\alpha, \beta$ and $\theta, X_{(r)}(r=1,2, \cdots, n)$ are $r$ th order statistics obtained by arranging $X_{r}$ $(r=1,2, \cdots, n)$ in ascending order of magnitude, then the corresponding $p d f f_{r: n}(x ; \alpha, \beta, \theta)$ is obtained by

$$
f_{r: n}(x ; \alpha, \beta, \theta)=\frac{n !}{(r-1) !(n-r) !} f(x)[F(x)]^{r-1}[1-F(x)]^{n-r},
$$

where $f(x)$ and $F(x)$ are $p d f$ and $c d f$ of DTEIW distribution, respectively. Using the binomial series expansion of $[1-F(x)]^{n-r}$ in (12), we obtain

$$
f_{r: n}(x ; \alpha, \beta, \theta)=\sum_{t=0}^{n-r} \frac{n !(-1)^{t}}{(r-1) !(n-r-t) ! t !} f(x)[F(x)]^{r+t-1} .
$$


Using the binomial series expansion of $[F(x)]^{r+t-1}$ into (13) and after some algebraic manipulations, we have

$$
f_{r: n}(x ; \alpha, \beta, \theta)=\sum_{t=0}^{n-r} \sum_{k=0}^{r+t-1} \psi_{r, k, t, n}\left(\alpha, \beta, \theta, t_{0}\right) f(x ; \alpha, \beta,(r+t-k) \theta),
$$

where

$$
\begin{aligned}
& \psi_{r, k, t, n}\left(\alpha, \beta, \theta, t_{0}\right)=\left(\begin{array}{c}
r+t-1 \\
k
\end{array}\right) \frac{n !(-1)^{t+k} e^{-k \alpha \theta t_{0}^{-\beta}}}{(r-1) !(n-r-t) ! t !(r+t-k) T^{k}} \\
& T=e^{-\alpha \theta t_{1}{ }^{-\beta}}-e^{-\alpha \theta t_{0}-\beta} \text { and } f(x ; \alpha, \beta,(r+t-k) \theta) \text { is the pdf of DTEIW distribution with } \\
& \text { parameters } \alpha, \beta,(r+t-k) \theta, t_{0} \text { and } t_{1} \text {. }
\end{aligned}
$$

\section{ESTIMATION OF PARAMETERS OF DTEIW DISTRIBUTION}

In the following subsections, we briefly describe the estimation procedures maximum likelihood, least squares and weighted least squares to obtain the estimates of the unknown parameters $\alpha, \beta$ and $\theta$ of DTEIW distribution.

\subsection{Maximum Likelihood Estimators}

Let $\left\{X_{1}, X_{2}, \cdots, X_{n}\right\}$ be a random sample of size $n$ from the DTEIW distribution. Then the corresponding log-likelihood function $L(\alpha, \beta, \theta)$ can be written as

$$
\ln L(\alpha, \beta, \theta)=n \ln (\alpha \beta \theta)-n \ln \left(e^{-\alpha \theta t_{1}^{-\beta}}-e^{-\alpha \theta t_{0}{ }^{-\beta}}\right)-\alpha \theta \sum_{i=1}^{n} x_{i}^{-\beta}-(\beta+1) \sum_{i=1}^{n} \ln x_{i} .
$$

The maximum likelihood (ML) estimates of the unknown parameters $\alpha, \beta$ and $\theta$ are the solutions of the nonlinear equations

$$
\begin{aligned}
\frac{\partial \ln L(\alpha, \beta, \theta)}{\partial \alpha}= & \frac{n}{\alpha}+n \theta \frac{\left(t_{1}^{-\beta} e^{-\alpha \theta t_{1}-\beta}-t_{0}^{-\beta} e^{-\alpha \theta t_{0}}-\beta\right.}{e^{-\alpha \theta t_{1}-\beta}-e^{-\alpha \theta t_{0}}-\beta}-\theta \sum_{i=1}^{n} x_{i}^{-\beta}=0 \\
\frac{\partial \ln L(\alpha, \beta, \theta)}{\partial \beta}= & \frac{n}{\beta}+\alpha \theta \sum_{i=1}^{n} x_{i}^{-\beta} \ln x_{i}-\sum_{i=1}^{n} \ln x_{i} \\
& -n \alpha \theta \frac{\left(t_{1}^{-\beta} \ln t_{1} e^{-\alpha \theta t_{1}-\beta}-t_{0}^{-\beta} \ln t_{0} e^{-\alpha \theta t_{0}}-\beta\right.}{e^{-\alpha \theta t_{1}-\beta}-e^{-\alpha \theta t_{0}-\beta}}=0 \\
\frac{\partial \ln L(\alpha, \beta, \theta)}{\partial \theta}= & \frac{n}{\theta}+n \alpha \frac{\left(t_{1}^{-\beta} e^{-\alpha \theta t_{1}-\beta}-t_{0}^{-\beta} e^{-\alpha \theta t_{0}-\beta}\right)}{e^{-\alpha \theta t_{1}-\beta}-e^{-\alpha \theta t_{0}}-\beta}-\alpha \sum_{i=1}^{n} x_{i}^{-\beta}=0,
\end{aligned}
$$

which may be solved to obtain the estimates of $\alpha, \beta$ and $\theta$ using a numerical procedure because the equations (14)-(16) do not have explicit solutions.

Now we represent the asymptotic normality results to obtain the asymptotic variances of $\hat{\alpha}, \hat{\beta}$ and $\hat{\theta}$. Therefore, approximate confidence intervals can be constructed for the individual parameters as the results of the asymptotic normality. This may be given by

$$
\sqrt{n}((\alpha-\hat{\alpha}),(\beta-\hat{\beta}),(\theta-\hat{\theta})) \stackrel{d}{\rightarrow} N\left(0, I^{-1}(\alpha, \beta, \theta)\right),
$$

where $I^{-1}(\alpha, \beta, \theta)$ is the asymptotic variance-covariance matrix of the $M L$ estimates of $\alpha, \beta$ and $\theta$ parameters, $I(\alpha, \beta, \theta)$ is the Fisher information matrix, i.e., 
Aydın / Anadolu Univ. J. of Sci. and Technology B - Theo. Sci. 6 (1) - 2018

$$
I(\alpha, \beta, \theta)=\left[\begin{array}{lll}
-E\left(\frac{\partial^{2} \ln L}{\partial \alpha^{2}}\right) & -E\left(\frac{\partial^{2} \ln L}{\partial \alpha \partial \beta}\right) & -E\left(\frac{\partial^{2} \ln L}{\partial \alpha \partial \theta}\right) \\
-E\left(\frac{\partial^{2} \ln L}{\partial \beta \partial \alpha}\right) & -E\left(\frac{\partial^{2} \ln L}{\partial \beta^{2}}\right) & -E\left(\frac{\partial^{2} \ln L}{\partial \beta \partial \theta}\right) \\
-E\left(\frac{\partial^{2} \ln L}{\partial \theta \partial \alpha}\right) & -E\left(\frac{\partial^{2} \ln L}{\partial \theta \partial \beta}\right) & -E\left(\frac{\partial^{2} \ln L}{\partial \theta^{2}}\right)
\end{array}\right],
$$

which provides a lower bound for the standard errors of estimates of individual parameters. The elements of $I(\alpha, \beta, \theta)$ are obtained as follows

$$
\begin{aligned}
& E\left(\frac{\partial^{2} \ln L(\alpha, \beta, \theta)}{\partial \alpha^{2}}\right)=-\frac{n}{\alpha^{2}}-n \theta^{2} \frac{\left(t_{1}^{-2 \beta} e^{-\alpha \theta t_{1}-\beta}-t_{0}^{-2 \beta} e^{-\alpha \theta t_{0}-\beta}\right)}{e^{-\alpha \theta t_{1}-\beta}-e^{-\alpha \theta t_{0}-\beta}} \\
& +n \theta^{2} \frac{\left(t_{1}^{-\beta} e^{-\alpha \theta t_{1}-\beta}-t_{0}-\beta e^{-\alpha \theta t_{0}-\beta}\right)^{2}}{\left(e^{-\alpha \theta t_{1}-\beta}-e^{-\alpha \theta t_{0}-\beta}\right)^{2}} \text {. } \\
& E\left(\frac{\partial^{2} \ln L(\alpha, \beta, \theta)}{\partial \theta^{2}}\right)=-\frac{n}{\theta^{2}}-n \alpha^{2} \frac{\left(t_{1}^{-2 \beta} e^{-\alpha \theta t_{1}-\beta}-t_{0}^{-2 \beta} e^{-\alpha \theta t_{0}-\beta}\right)}{e^{-\alpha \theta t_{1}-\beta}-e^{-\alpha \theta t_{0}-\beta}} \\
& +n \alpha^{2} \frac{\left(t_{1}^{-\beta} e^{-\alpha \theta t_{1}-\beta}-t_{0}^{-\beta} e^{-\alpha \theta t_{0}-\beta}\right)^{2}}{\left(e^{-\alpha \theta t_{1}-\beta}-e^{-\alpha \theta t_{0}-\beta}\right)^{2}} \text {. } \\
& E\left(\frac{\partial^{2} \ln L(\alpha, \beta, \theta)}{\partial \beta^{2}}\right)=-\frac{n}{\beta^{2}}+n \alpha \theta \frac{\left(t_{1}^{-\beta} \ln ^{2} t_{1} e^{-\alpha \theta t_{1}-\beta}\left(1-\alpha \theta t_{1}^{-\beta}\right)-t_{0}^{-\beta} \ln ^{2} t_{0} e^{-\alpha \theta t_{0}-\beta}\left(1-\alpha \theta t_{0}^{-\beta}\right)\right)}{e^{-\alpha \theta t_{1}^{-\beta}}-e^{-\alpha \theta t_{0}-\beta}} \\
& +n \alpha^{2} \theta^{2} \frac{\left(t_{1}^{-\beta} \ln t_{1} e^{-\alpha \theta t_{1}-\beta}-t_{0}^{-\beta} \ln t_{0} e^{-\alpha \theta t_{0}-\beta}\right)^{2}}{\left(e^{-\alpha \theta t_{1}-\beta}-e^{-\alpha \theta t_{0}-\beta}\right)^{2}} \\
& -\frac{n \alpha \theta}{e^{-\alpha \theta t_{1}^{-\beta}}-e^{-\alpha \theta t_{0}-\beta}} \int_{t_{0}}^{t_{1}} x^{-\beta} \ln ^{2} x \alpha \theta \beta x^{-(\beta+1)} e^{-\alpha \theta x^{-\beta}} d x \\
& =-\frac{n}{\beta^{2}}+n \alpha \theta \frac{\left(t_{1}^{-\beta}\left(\ln t_{1}\right)^{2} e^{-\alpha \theta t_{1}-\beta}\left(1-\alpha \theta t_{1}^{-\beta}\right)-t_{0}^{-\beta}\left(\operatorname{lnt} t_{0}\right)^{2} e^{-\alpha \theta t_{0}^{-\beta}}\left(1-\alpha \theta t_{0}^{-\beta}\right)\right)}{e^{-\alpha \theta t_{1}^{-\beta}}-e^{-\alpha \theta t_{0}-\beta}} \\
& +n \alpha^{2} \theta^{2} \frac{\left(t_{1}^{-\beta} \ln t_{1} e^{-\alpha \theta t_{1}-\beta}-t_{0}^{-\beta} \ln t_{0} e^{-\alpha \theta t_{0}-\beta}\right)^{2}}{\left(e^{-\alpha \theta t_{1}-\beta}-e^{-\alpha \theta t_{0}-\beta}\right)^{2}} \\
& -\frac{n \beta^{-2}}{e^{-\alpha \theta t_{1}-\beta}-e^{-\alpha \theta t_{0}}-\beta} \sum_{k=0}^{\infty} \frac{(-1)^{k}(\alpha \theta)^{k+2} t_{0}{ }^{-k \beta}}{k !}\left(\frac{\ln ^{2} \alpha \theta t_{0}-\beta}{n+1}-\frac{2 \ln \alpha \theta t_{0}-\beta}{(n+1)^{2}}+\frac{2}{(n+1)^{3}}\right) \\
& +\frac{n \beta^{-2}}{e^{-\alpha \theta t_{1}-\beta}-e^{-\alpha \theta t_{0}}-\beta} \sum_{k=0}^{\infty} \frac{(-1)^{k}(\alpha \theta)^{k+2} t_{1}{ }^{-k \beta}}{k !}\left(\frac{\ln ^{2} \alpha \theta t_{1}-\beta}{n+1}-\frac{2 \ln \alpha \theta t_{1}^{-\beta}}{(n+1)^{2}}+\frac{2}{(n+1)^{3}}\right) \\
& +\frac{2 n \beta^{-2} \ln \alpha \theta}{e^{-\alpha \theta t_{1}-\beta}-e^{-\alpha \theta t_{0}-\beta}} \sum_{k=0}^{\infty} \frac{(-1)^{k}(\alpha \theta)^{k+2} t_{0}{ }^{-k \beta}}{k !}\left(\frac{\ln \alpha \theta t_{0}-\beta}{n+1}-\frac{1}{(n+1)^{2}}\right) \\
& -\frac{2 n \beta^{-2} \ln \alpha \theta}{e^{-\alpha \theta t_{1}-\beta}-e^{-\alpha \theta t_{0}}-\beta} \sum_{k=0}^{\infty} \frac{(-1)^{k}(\alpha \theta)^{k+2} t_{1}{ }^{-k \beta}}{k !}\left(\frac{\ln \alpha \theta t_{1}-\beta}{n+1}-\frac{1}{(n+1)^{2}}\right) \\
& -\frac{n \beta^{-2} \ln ^{2} \alpha \theta}{e^{-\alpha \theta t_{1}-\beta}-e^{-\alpha \theta t_{0}}{ }^{-\beta}} \sum_{k=0}^{\infty} \frac{(-1)^{k}(\alpha \theta)^{k+2}}{k ! k}\left(t_{0}{ }^{-k \beta}-t_{1}{ }^{-k \beta}\right) \text {. }
\end{aligned}
$$

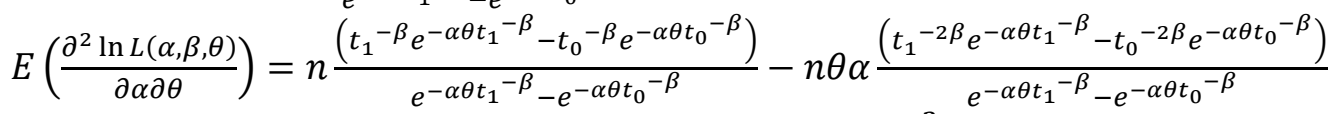

$$
\begin{aligned}
& +n \theta \alpha \frac{\left(t_{1}^{-\beta} e^{-\alpha \theta t_{1}^{-\beta}}-t_{0}^{-\beta} e^{-\alpha \theta t_{0}-\beta}\right)^{2}}{\left(e^{-\alpha \theta t_{1}^{-\beta}}-e^{-\alpha \theta t_{0}^{-\beta}}\right)^{2}} \\
& -\frac{n(\alpha \theta)^{-1}}{\left(e^{-\alpha \theta t_{1}^{-\beta}}-e^{-\alpha \theta t_{0}-\beta}\right)}\left(\gamma\left(2, \alpha \theta t_{0}^{-\beta}\right)-\gamma\left(2, \alpha \theta t_{1}^{-\beta}\right)\right) \text {. }
\end{aligned}
$$


Aydın / Anadolu Univ. J. of Sci. and Technology B - Theo. Sci. 6 (1) - 2018

$$
\begin{aligned}
& E\left(\frac{\partial^{2} \ln L(\alpha, \beta, \theta)}{\partial \alpha \partial \beta}\right)=-n \theta \frac{\left(t_{1}^{-\beta} \ln t_{1} e^{-\alpha \theta t_{1}-\beta}\left(1-\alpha \theta t_{1}^{-\beta}\right)-t_{0}^{-\beta} \ln t_{0} e^{-\alpha \theta t_{0}-\beta}\left(1-\alpha \theta t_{0}^{-\beta}\right)\right)}{e^{-\alpha \theta t_{1}-\beta}-e^{-\alpha \theta t_{0}}-\beta} \\
& -n \alpha \theta^{2} \frac{\left(t_{1}^{-\beta} \operatorname{lnt} t_{1} e^{-\alpha \theta t_{1}-\beta}-t_{0}{ }^{-\beta} \operatorname{lnt} t_{0} e^{-\alpha \theta t_{0}-\beta}\right)\left(t_{1}-\beta e^{-\alpha \theta t_{1}-\beta}-t_{0}-\beta e^{-\alpha \theta t_{0}-\beta}\right)}{\left(e^{-\alpha \theta t_{1}-\beta}-e^{-\alpha \theta t_{0}-\beta}\right)^{2}} \\
& +\frac{n \theta}{e^{-\alpha \theta t_{1}-\beta}-e^{-\alpha \theta t_{0}}-\beta} \int_{t_{0}}^{t_{1}} x^{-\beta} \ln x \alpha \theta \beta x^{-(\beta+1)} e^{-\alpha \theta x^{-\beta}} d x \\
& =-n \theta \frac{\left(t_{1}^{-\beta} \ln t_{1} e^{-\alpha \theta t_{1}-\beta}\left(1-\alpha \theta t_{1}^{-\beta}\right)-t_{0}^{-\beta} \ln t_{0} e^{-\alpha \theta t_{0}-\beta}\left(1-\alpha \theta t_{0}^{-\beta}\right)\right)}{e^{-\alpha \theta t_{1}^{-\beta}}-e^{-\alpha \theta t_{0}}{ }^{-\beta}} \\
& -n \alpha \theta^{2} \frac{\left(t_{1}^{-\beta} \ln t_{1} e^{-\alpha \theta t_{1}-\beta}-t_{0}^{-\beta} \ln t_{0} e^{-\alpha \theta t_{0}-\beta}\right)\left(t_{1}^{-\beta} e^{-\alpha \theta t_{1}-\beta}-t_{0}^{-\beta} e^{-\alpha \theta t_{0}-\beta}\right)}{\left(e^{-\alpha \theta t_{1}-\beta}-e^{-\alpha \theta t_{0}-\beta}\right)^{2}} \\
& +\frac{n}{\beta^{2}\left(e^{-\alpha \theta t_{1}}{ }^{-\beta}-e^{-\alpha \theta t_{0}}{ }^{-\beta}\right)} \sum_{k=0}^{\infty} \frac{(-1)^{k} \alpha^{k+1} \theta^{k+2} t_{0}{ }^{-k \beta}}{k !}\left(\frac{\ln \alpha \theta t_{0}-\beta}{n+1}-\frac{1}{(n+1)^{2}}\right) \\
& -\frac{n}{\beta^{2}\left(e^{-\alpha \theta t_{1}-\beta}-e^{-\alpha \theta t_{0}}{ }^{-\beta}\right)} \sum_{k=0}^{\infty} \frac{(-1)^{k} \alpha^{k+1} \theta^{k+2} t_{1}-k \beta}{k !}\left(\frac{\ln \alpha \theta t_{1}-\beta}{n+1}-\frac{1}{(n+1)^{2}}\right) \\
& -\frac{n \ln \alpha \theta}{\beta^{2}\left(e^{-\alpha \theta t_{1}{ }^{-\beta}-e^{-\alpha \theta t_{0}}-\beta}\right)} \sum_{k=0}^{\infty} \frac{(-1)^{k} \alpha^{k+1} \theta^{k+2}}{k ! k}\left(t_{0}{ }^{-k \beta}-t_{1}{ }^{-k \beta}\right) \text {. } \\
& E\left(\frac{\partial^{2} \ln L(\alpha, \beta, \theta)}{\partial \theta \partial \beta}\right)=-n \alpha \frac{\left(t_{1}^{-\beta} \ln t_{1} e^{-\alpha \theta t_{1}-\beta}\left(1-\alpha \theta t_{1}^{-\beta}\right)-t_{0}^{-\beta} \ln t_{0} e^{-\alpha \theta t_{0}-\beta}\left(1-\alpha \theta t_{0}^{-\beta}\right)\right)}{e^{-\alpha \theta t_{1}-\beta}-e^{-\alpha \theta t_{0}}-\beta} \\
& -n \alpha^{2} \theta \frac{\left(t_{1}^{-\beta} \operatorname{lnt}_{1} e^{-\alpha \theta t_{1}-\beta}-t_{0}{ }^{-\alpha \theta t_{1}-\beta}-e^{-\alpha \theta t_{0}} e^{-\alpha} e^{-\alpha \theta t_{0}}{ }^{-\beta}\right)\left(t_{1}{ }^{-\beta} e^{-\alpha \theta t_{1}-\beta}-t_{0}{ }^{-\beta} e^{-\alpha \theta t_{0}-\beta}\right)}{\left(e^{-\alpha \theta t_{1}-\beta}-e^{-\alpha \theta t_{0}-\beta}\right)^{2}} \\
& +\frac{n \alpha}{e^{-\alpha \theta t_{1}^{-\beta}}-e^{-\alpha \theta t_{0}}-\beta} \int_{t_{0}}^{t_{1}} x^{-\beta} \ln x \alpha \theta \beta x^{-(\beta+1)} e^{-\alpha \theta x^{-\beta}} d x \\
& =-n \alpha \frac{\left(t_{1}^{-\beta} \ln t_{1} e^{-\alpha \theta t_{1}-\beta}\left(1-\alpha \theta t_{1}^{-\beta}\right)-t_{0}^{-\beta} \ln t_{0} e^{-\alpha \theta t_{0}-\beta}\left(1-\alpha \theta t_{0}^{-\beta}\right)\right)}{e^{-\alpha \theta t_{1}^{-\beta}}-e^{-\alpha \theta t_{0}}-\beta} \\
& -n \alpha^{2} \theta \frac{\left(t_{1}^{-\beta} \operatorname{lnt}_{1} e^{-\alpha \theta t_{1}-\beta}-t_{0}^{-\beta} \operatorname{lnt}_{0} e^{-\alpha \theta t_{0}-\beta}\right)\left(t_{1}^{-\beta} e^{-\alpha \theta t_{1}-\beta}-t_{0}{ }^{-\beta} e^{-\alpha \theta t_{0}-\beta}\right)}{\left(e^{-\alpha \theta t_{1}-\beta}-e^{-\alpha \theta t_{0}-\beta}\right)^{2}} \\
& +\frac{n}{\beta^{2}\left(e^{-\alpha \theta t_{1}-\beta}-e^{-\alpha \theta t_{0}}{ }^{-\beta}\right)} \sum_{k=0}^{\infty} \frac{(-1)^{k} \alpha^{k+2} \theta^{k+1} t_{0}{ }^{-k \beta}}{k !}\left(\frac{\ln \alpha \theta t_{0}-\beta}{n+1}-\frac{1}{(n+1)^{2}}\right) \\
& -\frac{n}{\beta^{2}\left(e^{-\alpha \theta t_{1}-\beta}-e^{-\alpha \theta t_{0}}{ }^{-\beta}\right)} \sum_{k=0}^{\infty} \frac{(-1)^{k} \alpha^{k+2} \theta^{k+1} t_{1}-k \beta}{k !}\left(\frac{\ln \alpha \theta t_{1}-\beta}{n+1}-\frac{1}{(n+1)^{2}}\right) \\
& -\frac{n \ln \alpha \theta}{\beta^{2}\left(e^{-\alpha \theta t_{1}-\beta}-e^{-\alpha \theta t_{0}}{ }^{-\beta}\right)} \sum_{k=0}^{\infty} \frac{(-1)^{k} \alpha^{k+2} \theta^{k+1}}{k ! k}\left(t_{0}{ }^{-k \beta}-t_{1}{ }^{-k \beta}\right) \text {. }
\end{aligned}
$$

\subsection{Least Squares Estimators}

In this section, we provide the estimators of unknown parameters using the least squares $(L S)$ method which was originally proposed by Swain et al. [24]. Let $\left\{X_{1}, X_{2}, \cdots, X_{n}\right\}$ be a random sample of size $n$ from the DTEIW distribution with parameters $\alpha, \beta$ and $\theta$. Then the $L S$ estimators of parameters $\alpha, \beta$ and $\theta$ are obtained by minimizing the function

$$
G(\alpha, \beta, \theta)=\sum_{i=1}^{n}\left(F\left(x_{(i)}\right)-u_{i}\right)^{2},
$$


where $F\left(x_{(i)}\right)=\frac{e^{-\alpha \theta x_{(i)}}-\beta}{e^{-\alpha \theta t_{1}-\beta}-e^{-\alpha \theta t_{0}}-\beta}$ is the distribution function of the ordered random variables. Additionally, $u_{i}$ is used as an estimate of the $c d f$ in (3) for the $i$ th order statistics and is generally taken as $\frac{i}{n+1}$.

Then we obtain the normal equations as follows

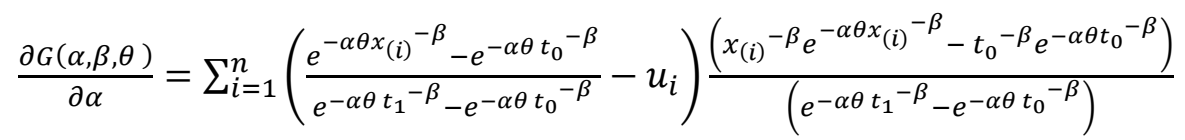

$$
\begin{aligned}
& -\sum_{i=1}^{n}\left(\frac{e^{-\alpha \theta x_{(i)}-\beta}-e^{-\alpha \theta t_{0}-\beta}}{e^{-\alpha \theta t_{1}-\beta}-e^{-\alpha \theta t_{0}-\beta}}-u_{i}\right) \frac{\left(t_{1}^{-\beta} e^{-\alpha \theta t_{1}-\beta}-t_{0}^{-\beta} e^{-\alpha \theta t_{0}-\beta}\right)\left(e^{-\alpha \theta x_{(i)}-\beta}-e^{-\alpha \theta t_{0}-\beta}\right)}{\left(e^{-\alpha \theta t_{1}-\beta}-e^{-\alpha \theta t_{0}}{ }^{-\beta}\right)^{2}}=0
\end{aligned}
$$

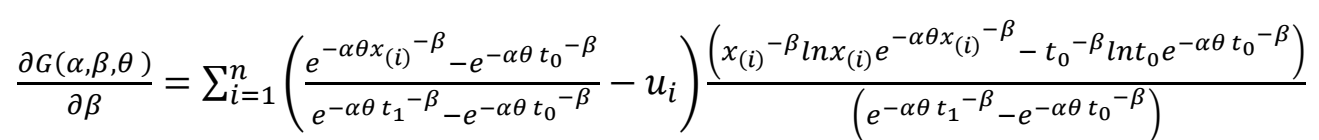

$$
\begin{aligned}
& -\sum_{i=1}^{n}\left(\frac{e^{-\alpha \theta x_{(i)}}-\beta}{e^{-\alpha \theta t_{1}-\beta}-e^{-\alpha \theta t_{0}}-\beta}-u_{i}\right) \frac{\left(t_{1}^{-\beta} t_{0}^{-\beta} \ln t_{1} e^{-\alpha \theta t_{1}}{ }^{-\beta}-t_{0}^{-\beta} \ln t_{0} e^{-\alpha \theta t_{0}}{ }^{-\beta}\right)\left(e^{-\alpha \theta x_{(i)}}-\beta-e^{-\alpha \theta t_{0}-\beta}\right)}{\left(e^{-\alpha \theta t_{1}-\beta}-e^{-\alpha \theta t_{0}}{ }^{-\beta}\right)^{2}}=0
\end{aligned}
$$

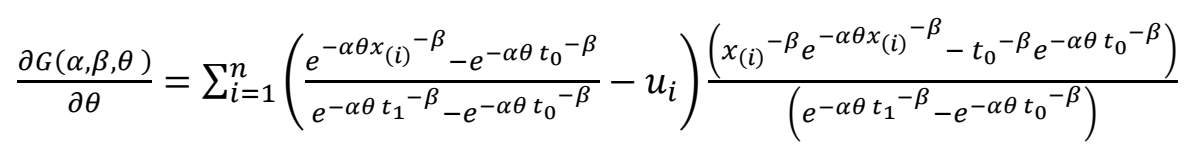

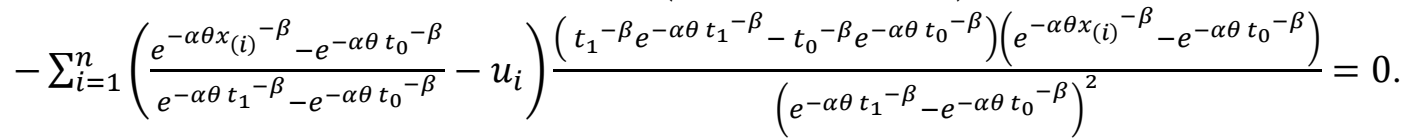

Here, $X_{(i)}(i=1,2, \cdots, n)$ are $i$ th order statistics and $x_{(i)}(i=1,2, \cdots, n)$ are ordered observations. The equations given in (20)-(22) can only be solved by iterative methods to obtain the $L S$ estimates of $\alpha, \beta$ and $\theta$.

\subsection{Weighted Least Squares Estimators}

The weighted least squares (WLS) (Swain et al. [24]) estimators of the unknown parameters can be obtained by minimizing the weighted sum of squares

$$
G_{w}(\alpha, \beta, \theta)=\sum_{i=1}^{n} w_{i}\left(F\left(x_{(i)}\right)-u_{i}\right)^{2},
$$

where $F\left(x_{(i)}\right)=\frac{e^{-\alpha \theta x_{(i)}-\beta}-e^{-\alpha \theta t_{0}}-\beta}{e^{-\alpha \theta t_{1}-\beta}-e^{-\alpha \theta t_{0}}-\beta}$. Then we have following normal equations

$$
\begin{aligned}
& \frac{\partial G_{w}(\alpha, \beta, \theta)}{\partial \alpha}=\sum_{i=1}^{n} w_{i}\left(\frac{e^{-\alpha \theta x_{(i)}}-\beta}{e^{-\alpha \theta t_{1}-\beta}-e^{-\alpha \theta t_{0}}-\beta}-u_{i}\right) \frac{\left.\left(x_{(i)}\right)^{-\beta} e^{-\alpha \theta x_{(i)}}-\beta-t_{0}^{-\beta} e^{-\alpha \theta t_{0}-\beta}\right)}{\left(e^{-\alpha \theta t_{1}-\beta}-e^{-\alpha \theta t_{0}}-\beta\right.}
\end{aligned}
$$

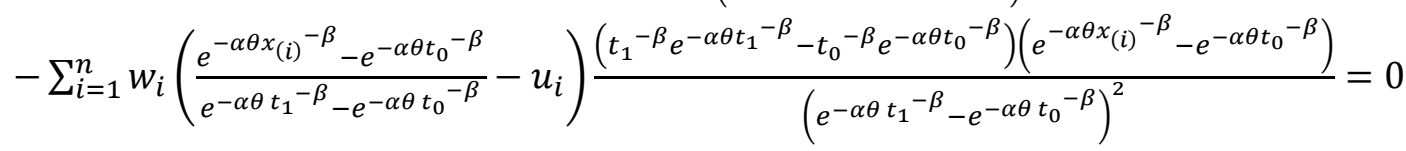




$$
\begin{aligned}
& \frac{\partial G_{w}(\alpha, \beta, \theta)}{\partial \beta}=\sum_{i=1}^{n} w_{i}\left(\frac{e^{-\alpha \theta x_{(i)}}-\beta}{e^{-\alpha \theta t_{1}^{-\beta}}-e^{-\alpha \theta t_{0}}-\beta}-u_{i}\right) \frac{\left(x_{(i)}^{-\beta} \ln x_{(i)} e^{-\alpha \theta x_{(i)}-\beta}-t_{0}^{-\beta} \ln t_{0} e^{-\alpha \theta t_{0}-\beta}\right)}{\left(e^{-\alpha \theta t_{1}^{-\beta}}-e^{-\alpha \theta t_{0}}{ }^{-\beta}\right)}
\end{aligned}
$$

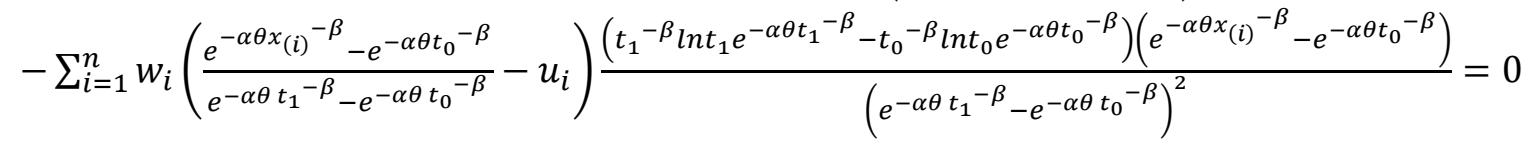

$$
\begin{aligned}
& \left.\frac{\partial G_{w}(\alpha, \beta, \theta)}{\partial \theta}=\sum_{i=1}^{n} w_{i}\left(\frac{e^{-\alpha \theta x_{(i)}}-\beta}{e^{-\alpha \theta t_{1}^{-\beta}}-e^{-\alpha \theta t_{0}}-\beta}-u_{i}\right) \frac{\left(x_{(i)}^{-\beta} e^{-\alpha \theta x_{(i)}-\beta}-t_{0}^{-\beta} e^{-\alpha \theta t_{0}-\beta}\right)}{\left(e^{-\alpha \theta t_{1}-\beta}-e^{-\alpha \theta t_{0}}-\beta\right.}\right) \\
& \left.-\sum_{i=1}^{n} w_{i}\left(\frac{e^{-\alpha \theta x_{(i)}}-e^{-\alpha \theta t_{0}}-\beta}{e^{-\alpha \theta t_{1}^{-\beta}}-e^{-\alpha \theta t_{0}}-\beta}-u_{i}\right) \frac{\left(t_{1}^{-\beta} e^{-\alpha \theta t_{1}-\beta}-t_{0}^{-\beta} e^{-\alpha \theta t_{0}-\beta}\right)\left(e^{-\alpha \theta x_{(i)}-\beta}-e^{-\alpha \theta t_{0}-\beta}\right)}{\left(e^{-\alpha \theta t_{1}-\beta}-e^{-\alpha \theta t_{0}}-\beta\right.}\right)^{2}=0,
\end{aligned}
$$

where $w_{i}=\frac{1}{\operatorname{Var}\left(F\left(x_{(i)}\right)\right)}$ and $\operatorname{Var}\left(F\left(x_{(i)}\right)\right)=\frac{i(n-i+1)}{(n+1)^{2}(n+2)}$, see Johnson et al. [13]. The iterative method is applied to solve the nonlinear equations given in (23)-(25) simultaneously to obtain the WLS estimates $\hat{\alpha}_{W L S}, \hat{\beta}_{W L S}$ and $\hat{\theta}_{W L S}$ of $\alpha, \beta$ and $\theta$, respectively.

\section{SIMULATION STUDY}

This section focuses on the performances of different estimation methods for the unknown parameters of DTEIW distribution that are presented in Section 3. A computer program in Matlab is designed and conducted for extended simulation studies. In order to evaluate their performances, we use bias and root mean square error (RMSE) comparison criteria, which are given by the following formulas

and

$$
\operatorname{Bias}(\widehat{\phi})=\frac{1}{r} \sum_{i=1}^{r}\left(\phi-\widehat{\phi}_{i}\right)
$$

$$
\operatorname{RMSE}(\hat{\phi})=\sqrt{\frac{1}{r} \sum_{i=1}^{r}\left(\phi-\hat{\phi}_{i}\right)^{2}}
$$

Here, $r$ is number of replications and $\hat{\phi}_{i}$ is the estimate of any unknown parameter $\phi$ in the $i$ th replication.

In this study, we use the inverse transformation method to generate random numbers having the DTEIW distribution:

$$
x=\left(-\frac{1}{\alpha \theta} \ln \left(u T+e^{-\alpha \theta t_{0}^{-\beta}}\right)\right)^{-1 / \beta},
$$

where $T=e^{-\alpha \theta t_{1}^{-\beta}}-e^{-\alpha \theta t_{0}^{-\beta}}$ and $u$ has uniform distribution as $u \sim U(0,1)$.

For comparison, 5000 replications of different sample sizes $n=10,20,50,100$ are performed for certain selected parameter values $(\alpha, \beta, \theta)=(0.5,1,1),(0.5,1,2),(0.5,2,1),(0.5,2,2),(1,1,1),(1$, $1,2),(1,2,1),(1,2,2),(2,1,1),(2,1,2),(2,2,1)$ and $(2,2,2)$. Furthermore, we chose truncation points $t_{0}$ and $t_{1}$ so as $\hat{t}_{0}=x_{0.05}$ (where $x_{0.05}$ is $q$ th quantile of $E I W$ distribution for $q=0.05$ ) and $\hat{t}_{1}=x_{0.95}$ (where $x_{0.95}$ is is $q$ th quantile of $E I W$ distribution for $q=0.95$ ), respectively; see Kantar and Usta [16]. Simulation results showing the performances of the estimators of unknown parameters are given in Tables 1-3. From tables 1-3, the following conclusions can be drawn:

In terms of the bias, 
(i) For $\alpha$ : When the sample size is $n=10, M L$ has smallest bias among the others in almost all cases. However, when $n=100$, the $L S$ method shows the best performance in many cases. It is also observed that the biases of considered estimation methods decrease as sample size increases. Finally, it can said that the $M L, L S$ and the $W L S$ estimators overestimate when shape parameter $\alpha>$ 0.5 and sample size $n=10$.

(ii) For $\beta$ : When the sample size is $n=10$, the $W L S$ outperforms other estimation methods in almost all cases. The estimator with the smallest bias is $M L$ for many sample sizes; $n=20$, in particular.

(iii) For $\theta$ : The $M L$ estimator performs best among all considered estimation methods in many cases and all sample sizes (except for sample size $n=20$ ). Additionally, for $n=10$, the $M L, L S$ and $W L S$ estimators overestimate in certain cases.

Table 1. Simulated Bias and RMSE values for the estimator of $\alpha$.

\begin{tabular}{|c|c|c|c|c|c|c|c|}
\hline \multirow[b]{2}{*}{$n$} & & \multicolumn{3}{|c|}{ Bias } & \multicolumn{3}{|c|}{$R M S E$} \\
\hline & & $\hat{\alpha}_{M L}$ & $\hat{\alpha}_{L S}$ & $\hat{\alpha}_{W L S}$ & $\hat{\alpha}_{M L}$ & $\hat{\alpha}_{L S}$ & $\hat{\alpha}_{W L S}$ \\
\hline \multirow[t]{12}{*}{10} & $(0.5,1,1)$ & -0.0434 & -0.8175 & -0.6959 & 1.9913 & 7.3326 & 6.5208 \\
\hline & $(0.5,1,2)$ & -0.6159 & -0.6896 & -0.8216 & 5.0156 & 5.8222 & 6.5731 \\
\hline & $(0.5,2,1)$ & -0.1856 & -0.6659 & -0.8131 & 6.1914 & 6.9327 & 10.2885 \\
\hline & $(0.5,2,2)$ & -0.4964 & -0.7504 & -0.7803 & 4.8905 & 5.6882 & 5.9276 \\
\hline & $(1,1,1)$ & -1.2253 & -1.3909 & -1.1973 & 10.1212 & 12.5330 & 11.6757 \\
\hline & $(1,1,2)$ & -0.6211 & -0.7759 & -0.6091 & 4.5042 & 6.1067 & 4.2272 \\
\hline & $(1,2,1)$ & -1.0564 & -1.5684 & -1.4436 & 9.4962 & 14.1033 & 10.8304 \\
\hline & $(1,2,2)$ & -0.6238 & -0.7210 & -0.6665 & 4.5007 & 4.5025 & 4.7621 \\
\hline & $(2,1,1)$ & -1.2337 & -1.2903 & -1.2380 & 10.7823 & 9.5260 & 9.5087 \\
\hline & $(2,1,2)$ & -0.9162 & -0.8476 & -0.8341 & 2.6176 & 5.9427 & 5.9775 \\
\hline & $(2,2,1)$ & -1.2379 & -1.1799 & -1.3757 & 8.6717 & 8.0163 & 12.1037 \\
\hline & $(2,2,2)$ & -0.9493 & -1.1399 & -1.0573 & 3.9078 & 14.3413 & 9.7749 \\
\hline \multirow[t]{12}{*}{20} & $(0.5,1,1)$ & -0.0523 & -0.1263 & -0.1199 & 1.9809 & 2.1782 & 4.6679 \\
\hline & $(0.5,1,2)$ & -0.1220 & -0.1998 & -0.2110 & 1.3601 & 2.3411 & 4.0298 \\
\hline & $(0.5,2,1)$ & -0.0216 & -0.0880 & -0.1019 & 1.5781 & 1.5118 & 2.3555 \\
\hline & $(0.5,2,2)$ & -0.1889 & -0.1342 & -0.1180 & 2.2794 & 1.8244 & 1.6088 \\
\hline & $(1,1,1)$ & -0.2722 & -0.3816 & -0.2969 & 3.5033 & 5.1776 & 4.7319 \\
\hline & $(1,1,2)$ & -0.2084 & -0.1934 & -0.2061 & 1.3488 & 2.0028 & 2.1386 \\
\hline & $(1,2,1)$ & -0.2947 & -0.2525 & -0.3334 & 3.7405 & 3.5407 & 4.3716 \\
\hline & $(1,2,2)$ & -0.2708 & -0.1780 & -0.1723 & 1.9074 & 1.8700 & 1.4893 \\
\hline & $(2,1,1)$ & -0.4752 & -0.2260 & -0.2748 & 3.7068 & 1.1887 & 2.6695 \\
\hline & $(2,1,2)$ & -0.4323 & -0.2787 & -0.2499 & 1.3808 & 3.6876 & 2.3113 \\
\hline & $(2,2,1)$ & -0.3755 & -0.3108 & -0.3555 & 1.8265 & 3.0006 & 4.9833 \\
\hline & $(2,2,2)$ & -0.4396 & -0.2101 & -0.2918 & 1.1981 & 1.9276 & 3.7599 \\
\hline \multirow[t]{12}{*}{50} & $(0.5,1,1)$ & -0.0141 & -0.0281 & -0.0254 & 0.1046 & 0.3648 & 0.1216 \\
\hline & $(0.5,1,2)$ & -0.0321 & -0.0364 & -0.0335 & 0.0933 & 0.1117 & 0.2269 \\
\hline & $(0.5,2,1)$ & -0.0133 & -0.0278 & -0.0279 & 0.1030 & 0.1232 & 0.1778 \\
\hline & $(0.5,2,2)$ & -0.0330 & -0.0330 & -0.0331 & 0.0904 & 0.1429 & 0.1294 \\
\hline & $(1,1,1)$ & -0.0676 & -0.0712 & -0.0687 & 0.1664 & 0.2197 & 0.1842 \\
\hline & $(1,1,2)$ & -0.0962 & -0.0784 & -0.0803 & 0.2627 & 0.2509 & 0.2228 \\
\hline & $(1,2,1)$ & -0.0763 & -0.0650 & -0.0628 & 0.9691 & 0.2182 & 0.7833 \\
\hline & $(1,2,2)$ & -0.0932 & -0.0728 & -0.0732 & 0.1975 & 0.2527 & 0.2284 \\
\hline & $(2,1,1)$ & -0.1947 & -0.1393 & -0.1530 & 0.5562 & 0.6116 & 0.5989 \\
\hline & $(2,1,2)$ & -0.2570 & -0.1257 & -0.1589 & 0.7015 & 0.6978 & 0.6508 \\
\hline & $(2,2,1)$ & -0.1859 & -0.1316 & -0.1407 & 0.3987 & 0.4841 & 0.4454 \\
\hline & $(2,2,2)$ & -0.2481 & -0.1152 & -0.1505 & 0.8537 & 0.6830 & 0.6122 \\
\hline \multirow[t]{12}{*}{100} & $(0.5,1,1)$ & -0.0184 & -0.0272 & -0.0253 & 0.0488 & 0.0543 & 0.0497 \\
\hline & $(0.5,1,2)$ & -0.0280 & -0.0247 & -0.0251 & 0.0465 & 0.0586 & 0.0516 \\
\hline & $(0.5,2,1)$ & -0.0187 & -0.0263 & -0.0259 & 0.0454 & 0.0961 & 0.0475 \\
\hline & $(0.5,2,2)$ & -0.0282 & -0.0256 & -0.0265 & 0.0456 & 0.0560 & 0.0506 \\
\hline & $(1,1,1)$ & -0.0557 & -0.0477 & -0.0515 & 0.1001 & 0.1170 & 0.1054 \\
\hline & $(1,1,2)$ & -0.0707 & -0.0604 & -0.0632 & 0.1323 & 0.1564 & 0.1489 \\
\hline & $(1,2,1)$ & -0.0557 & -0.0511 & -0.0532 & 0.0933 & 0.1175 & 0.1055 \\
\hline & $(1,2,2)$ & -0.0697 & -0.0570 & -0.0605 & 0.1291 & 0.1627 & 0.1514 \\
\hline & $(2,1,1)$ & -0.1381 & -0.1190 & -0.1237 & 0.2712 & 0.3287 & 0.2969 \\
\hline & $(2,1,2)$ & -0.1804 & -0.1159 & -0.1349 & 0.3614 & 0.4468 & 0.4017 \\
\hline & $(2,2,1)$ & -0.1460 & -0.1276 & -0.1358 & 0.2695 & 0.3262 & 0.3057 \\
\hline & $(2,2,2)$ & -0.1807 & -0.0969 & -0.1248 & 0.3701 & 0.4433 & 0.4174 \\
\hline
\end{tabular}


Aydın / Anadolu Univ. J. of Sci. and Technology B - Theo. Sci. 6(1) - 2018

Table 2. Simulated Bias and RMSE values for the estimator of $\beta$.

\begin{tabular}{|c|c|c|c|c|c|c|c|}
\hline \multirow[b]{2}{*}{$n$} & & \multicolumn{3}{|c|}{ Bias } & \multicolumn{3}{|c|}{ RMSE } \\
\hline & & $\hat{\beta}_{M L}$ & $\hat{\beta}_{L S}$ & $\hat{\beta}_{W L S}$ & $\hat{\beta}_{M L}$ & $\hat{\beta}_{L S}$ & $\hat{\beta}_{W L S}$ \\
\hline \multirow[t]{12}{*}{10} & $(0.5,1,1)$ & -0.1179 & 0.1358 & 0.1205 & 0.5818 & 0.6490 & 0.6265 \\
\hline & $(0.5,1,2)$ & -0.1392 & 0.1126 & 0.1026 & 0.5938 & 0.6247 & 0.6026 \\
\hline & $(0.5,2,1)$ & -0.2602 & 0.2373 & 0.2134 & 1.1729 & 1.2646 & 1.2154 \\
\hline & $(0.5,2,2)$ & -0.2485 & 0.2431 & 0.2223 & 1.1500 & 1.2928 & 1.2347 \\
\hline & $(1,1,1)$ & -0.1387 & 0.1105 & 0.1002 & 0.5852 & 0.6509 & 0.6099 \\
\hline & $(1,1,2)$ & -0.1202 & 0.1379 & 0.1262 & 0.5700 & 0.6380 & 0.6109 \\
\hline & $(1,2,1)$ & -0.2420 & 0.2586 & 0.2358 & 1.1798 & 1.2984 & 1.2514 \\
\hline & $(1,2,2)$ & -0.2763 & 0.2279 & 0.1977 & 1.1660 & 1.2555 & 1.2144 \\
\hline & $(2,1,1)$ & -0.1293 & 0.1188 & 0.1070 & 0.5804 & 0.6477 & 0.6164 \\
\hline & $(2,1,2)$ & -0.1389 & 0.1156 & 0.1066 & 0.5901 & 0.6368 & 0.6062 \\
\hline & $(2,2,1)$ & -0.2633 & 0.2659 & 0.2391 & 1.1705 & 1.2489 & 1.1967 \\
\hline & $(2,2,2)$ & -0.2674 & 0.2371 & 0.2121 & 1.1552 & 1.2844 & 1.2288 \\
\hline \multirow[t]{12}{*}{20} & $(0.5,1,1)$ & -0.0498 & 0.0803 & 0.0576 & 0.3880 & 0.4341 & 0.4097 \\
\hline & $(0.5,1,2)$ & -0.0426 & 0.0956 & 0.0680 & 0.3887 & 0.4389 & 0.4132 \\
\hline & $(0.5,2,1)$ & -0.0934 & 0.1861 & 0.1346 & 0.7650 & 0.8613 & 0.8132 \\
\hline & $(0.5,2,2)$ & -0.0813 & 0.1828 & 0.1313 & 0.7640 & 0.8579 & 0.8128 \\
\hline & $(1,1,1)$ & -0.0402 & 0.0929 & 0.0695 & 0.3862 & 0.4335 & 0.4115 \\
\hline & $(1,1,2)$ & -0.0420 & 0.0905 & 0.0662 & 0.3867 & 0.4363 & 0.4125 \\
\hline & $(1,2,1)$ & -0.0613 & 0.1980 & 0.1480 & 0.7680 & 0.8731 & 0.8249 \\
\hline & $(1,2,2)$ & -0.0765 & 0.1886 & 0.1364 & 0.7651 & 0.8627 & 0.8193 \\
\hline & $(2,1,1)$ & -0.0389 & 0.0966 & 0.0714 & 0.3730 & 0.4239 & 0.4003 \\
\hline & $(2,1,2)$ & -0.0435 & 0.0870 & 0.0625 & 0.3817 & 0.4356 & 0.4135 \\
\hline & $(2,2,1)$ & -0.0801 & 0.1834 & 0.1320 & 0.7521 & 0.8647 & 0.8104 \\
\hline & $(2,2,2)$ & -0.0755 & 0.1788 & 0.1349 & 0.7683 & 0.8702 & 0.8290 \\
\hline \multirow[t]{12}{*}{50} & $(0.5,1,1)$ & -0.0123 & 0.0403 & 0.0215 & 0.2263 & 0.2552 & 0.2367 \\
\hline & $(0.5,1,2)$ & -0.0135 & 0.0361 & 0.0190 & 0.2316 & 0.2568 & 0.2405 \\
\hline & $(0.5,2,1)$ & -0.0244 & 0.0772 & 0.0416 & 0.4536 & 0.5109 & 0.4747 \\
\hline & $(0.5,2,2)$ & -0.0242 & 0.0778 & 0.0421 & 0.4492 & 0.5026 & 0.4661 \\
\hline & $(1,1,1)$ & -0.0189 & 0.0332 & 0.0149 & 0.2313 & 0.2571 & 0.2397 \\
\hline & $(1,1,2)$ & -0.0135 & 0.0361 & 0.0195 & 0.2329 & 0.2594 & 0.2425 \\
\hline & $(1,2,1)$ & -0.0378 & 0.0621 & 0.0275 & 0.4473 & 0.5025 & 0.4664 \\
\hline & $(1,2,2)$ & -0.0256 & 0.0800 & 0.0431 & 0.4605 & 0.5124 & 0.4775 \\
\hline & $(2,1,1)$ & -0.0130 & 0.0356 & 0.0190 & 0.2265 & 0.2535 & 0.2354 \\
\hline & $(2,1,2)$ & -0.0161 & 0.0367 & 0.0181 & 0.2298 & 0.2559 & 0.2391 \\
\hline & $(2,2,1)$ & -0.0331 & 0.0633 & 0.0311 & 0.4555 & 0.5050 & 0.4720 \\
\hline & $(2,2,2)$ & -0.0241 & 0.0829 & 0.0450 & 0.4512 & 0.5103 & 0.4730 \\
\hline \multirow[t]{12}{*}{100} & $(0.5,1,1)$ & -0.0057 & 0.0205 & 0.0088 & 0.1534 & 0.1674 & 0.1568 \\
\hline & $(0.5,1,2)$ & -0.0084 & 0.0164 & 0.0058 & 0.1513 & 0.1651 & 0.1544 \\
\hline & $(0.5,2,1)$ & -0.0070 & 0.0432 & 0.0212 & 0.3069 & 0.3354 & 0.3133 \\
\hline & $(0.5,2,2)$ & -0.0123 & 0.0370 & 0.0153 & 0.3007 & 0.3324 & 0.3097 \\
\hline & $(1,1,1)$ & -0.0082 & 0.0178 & 0.0063 & 0.1570 & 0.1711 & 0.1606 \\
\hline & $(1,1,2)$ & -0.0097 & 0.0150 & 0.0041 & 0.1541 & 0.1677 & 0.1575 \\
\hline & $(1,2,1)$ & -0.0117 & 0.0382 & 0.0154 & 0.3066 & 0.3374 & 0.3147 \\
\hline & $(1,2,2)$ & -0.0238 & 0.0235 & 0.0026 & 0.3025 & 0.3284 & 0.3084 \\
\hline & $(2,1,1)$ & -0.0045 & 0.0209 & 0.0101 & 0.1572 & 0.1696 & 0.1595 \\
\hline & $(2,1,2)$ & -0.0048 & 0.0204 & 0.0091 & 0.1544 & 0.1704 & 0.1579 \\
\hline & $(2,2,1)$ & -0.0145 & 0.0337 & 0.0126 & 0.3074 & 0.3333 & 0.3125 \\
\hline & $(2,2,2)$ & -0.0119 & 0.0399 & 0.0168 & 0.3041 & 0.3328 & 0.3108 \\
\hline
\end{tabular}


Aydın / Anadolu Univ. J. of Sci. and Technology B - Theo. Sci. 6 (1) - 2018

Table 3. Simulated Bias and RMSE values for the estimator of $\theta$.

\begin{tabular}{|c|c|c|c|c|c|c|c|}
\hline \multirow[b]{2}{*}{$n$} & & \multicolumn{3}{|c|}{ Bias } & \multicolumn{3}{|c|}{ RMSE } \\
\hline & & $\hat{\theta}_{M L}$ & $\hat{\theta}_{L S}$ & $\hat{\theta}_{W L S}$ & $\hat{\theta}_{M L}$ & $\hat{\theta}_{L S}$ & $\hat{\theta}_{W L S}$ \\
\hline \multirow[t]{12}{*}{10} & $(0.5,1,1)$ & 0.1273 & -1.5119 & -1.0727 & 4.1647 & 249.0086 & 102.3525 \\
\hline & $(0.5,1,2)$ & -1.4715 & -1.1467 & -0.9515 & 317.2880 & 265.5634 & 202.1522 \\
\hline & $(0.5,2,1)$ & 0.0900 & -1.1264 & -1.1266 & 16.2702 & 144.4426 & 205.5666 \\
\hline & $(0.5,2,2)$ & -1.3814 & -1.1976 & -1.5097 & 302.8875 & 227.4384 & 317.3447 \\
\hline & $(1,1,1)$ & -0.9316 & -0.5419 & -0.5345 & 121.6830 & 82.4167 & 65.6701 \\
\hline & $(1,1,2)$ & -0.2436 & -0.8133 & -0.8315 & 23.4619 & 191.3808 & 153.2735 \\
\hline & $(1,2,1)$ & -0.8264 & -0.8264 & -0.8179 & 92.3538 & 114.7585 & 86.9253 \\
\hline & $(1,2,2)$ & -0.5349 & -0.7509 & -0.8696 & 142.3820 & 97.0394 & 110.9891 \\
\hline & $(2,1,1)$ & -0.2009 & -0.2942 & -0.4025 & 13.2198 & 24.3896 & 39.9315 \\
\hline & $(2,1,2)$ & -0.4776 & -0.7482 & -0.7402 & 35.1916 & 66.7215 & 75.3803 \\
\hline & $(2,2,1)$ & -0.1802 & -0.4245 & -0.2672 & 8.7655 & 60.0023 & 23.4355 \\
\hline & $(2,2,2)$ & -0.3925 & -1.1591 & -0.8393 & 13.9415 & 326.1629 & 126.5488 \\
\hline \multirow[t]{12}{*}{20} & $(0.5,1,1)$ & 0.0489 & -0.0384 & -0.0068 & 7.1913 & 16.9484 & 16.2856 \\
\hline & $(0.5,1,2)$ & -0.1924 & 0.1018 & 0.1585 & 82.6297 & 48.4469 & 34.5928 \\
\hline & $(0.5,2,1)$ & 0.1237 & -0.2157 & 0.0046 & 0.6614 & 38.9459 & 14.6674 \\
\hline & $(0.5,2,2)$ & -0.2634 & 0.0078 & 0.1804 & 64.2138 & 98.6992 & 25.8014 \\
\hline & $(1,1,1)$ & -0.1708 & 0.1213 & 0.1417 & 22.7056 & 6.9417 & 2.2533 \\
\hline & $(1,1,2)$ & 0.0697 & 0.2050 & 0.0825 & 15.0391 & 7.8711 & 32.1653 \\
\hline & $(1,2,1)$ & -0.2205 & 0.1793 & 0.1066 & 28.9364 & 2.1896 & 4.7714 \\
\hline & $(1,2,2)$ & 0.0072 & 0.1687 & 0.1756 & 16.8029 & 21.7449 & 23.2211 \\
\hline & $(2,1,1)$ & -0.0087 & 0.1414 & 0.0925 & 6.3389 & 1.8392 & 1.8756 \\
\hline & $(2,1,2)$ & -0.0412 & 0.0330 & -0.0052 & 20.9524 & 16.4251 & 16.1111 \\
\hline & $(2,2,1)$ & 0.0740 & 0.0918 & 0.1038 & 0.7144 & 4.0854 & 2.7938 \\
\hline & $(2,2,2)$ & 0.0437 & 0.0264 & 0.0128 & 1.5967 & 8.6550 & 17.7638 \\
\hline \multirow[t]{12}{*}{50} & $(0.5,1,1)$ & 0.0616 & 0.0901 & 0.0839 & 0.0538 & 0.3179 & 0.0639 \\
\hline & $(0.5,1,2)$ & 0.1518 & 0.2136 & 0.1946 & 0.1946 & 0.3492 & 0.3200 \\
\hline & $(0.5,2,1)$ & 0.0613 & 0.1054 & 0.0868 & 0.0551 & 0.2050 & 0.0717 \\
\hline & $(0.5,2,2)$ & 0.1584 & 0.2076 & 0.1798 & 0.2579 & 0.3882 & 0.2546 \\
\hline & $(1,1,1)$ & 0.0787 & 0.1053 & 0.0958 & 0.0466 & 0.0913 & 0.0790 \\
\hline & $(1,1,2)$ & 0.1306 & 0.1964 & 0.1701 & 0.3164 & 0.3938 & 0.3567 \\
\hline & $(1,2,1)$ & 0.0679 & 0.0950 & 0.0829 & 0.0579 & 0.0765 & 0.1582 \\
\hline & $(1,2,2)$ & 0.1436 & 0.2044 & 0.1698 & 0.2561 & 0.4334 & 0.3385 \\
\hline & $(2,1,1)$ & 0.0686 & 0.0914 & 0.0720 & 0.0754 & 0.0972 & 0.1924 \\
\hline & $(2,1,2)$ & 0.0865 & 0.1073 & 0.0997 & 0.4393 & 0.5152 & 0.4381 \\
\hline & $(2,2,1)$ & 0.0652 & 0.0828 & 0.0726 & 0.0607 & 0.0922 & 0.0796 \\
\hline & $(2,2,2)$ & 0.0981 & 0.1191 & 0.1054 & 0.3768 & 0.5118 & 0.4742 \\
\hline \multirow[t]{12}{*}{100} & $(0.5,1,1)$ & 0.0432 & 0.0572 & 0.0536 & 0.0128 & 0.0176 & 0.0154 \\
\hline & $(0.5,1,2)$ & 0.1142 & 0.1196 & 0.1119 & 0.0827 & 0.0962 & 0.0814 \\
\hline & $(0.5,2,1)$ & 0.0464 & 0.0617 & 0.0577 & 0.0136 & 0.0209 & 0.0165 \\
\hline & $(0.5,2,2)$ & 0.1125 & 0.1236 & 0.1163 & 0.0642 & 0.0949 & 0.0796 \\
\hline & $(1,1,1)$ & 0.0596 & 0.0638 & 0.0615 & 0.0176 & 0.0277 & 0.0214 \\
\hline & $(1,1,2)$ & 0.1057 & 0.1271 & 0.1130 & 0.0993 & 0.1613 & 0.1328 \\
\hline & $(1,2,1)$ & 0.0558 & 0.0609 & 0.0576 & 0.0163 & 0.0231 & 0.0200 \\
\hline & $(1,2,2)$ & 0.1074 & 0.1177 & 0.1066 & 0.1019 & 0.1579 & 0.1293 \\
\hline & $(2,1,1)$ & 0.0578 & 0.0694 & 0.0626 & 0.0278 & 0.0415 & 0.0354 \\
\hline & $(2,1,2)$ & 0.0962 & 0.1021 & 0.0877 & 0.1978 & 0.2659 & 0.2352 \\
\hline & $(2,2,1)$ & 0.0546 & 0.0645 & 0.0589 & 0.0250 & 0.0412 & 0.0338 \\
\hline & $(2,2,2)$ & 0.1003 & 0.0861 & 0.0811 & 0.1869 & 0.2635 & 0.2258 \\
\hline
\end{tabular}

In terms of the RMSE,

(i) For $\alpha$ : The $M L$ estimator shows the best performance among the others in many cases, even for small sample sizes.

(ii) For $\beta$ : The $M L$ estimator gives the best results for estimating the scale parameter for all cases and all sample sizes. 
(iii) For $\theta$ : When sample sizes are moderate $(n=50)$ and large $(n=100)$, the performance of the $M L$ estimator is the best with the smallest $R M S E$ values in many cases. However, we observe that $M L$, $L S$ and the $W L S$ estimates have quite large $R M S E$ values for small sample sizes.

\section{DATA ANALYSIS}

In this section, we present the comparison analysis of the DTEIW and EIW distributions applying to a real data set. The data in Table 4 consists of the survival in months of 20 acute myeloid leukaemia patients reported in Afify et al. [1].

Table 4. Survival times in months of 20 acute myeloid leukaemia patients.

\begin{tabular}{llllllllll}
\hline 2.226 & 2.113 & 3.631 & 2.473 & 2.720 & 2.050 & 2.061 & 3.915 & 0.871 & 1.548 \\
2.746 & 1.972 & 2.265 & 1.200 & 2.967 & 2.808 & 1.079 & 2.353 & 0.726 & 1.958 \\
\hline
\end{tabular}

The data is modelled by using the DTEIW and EIW distributions. The Kolmogorov-Smirnov $(K-S)$ test is used to assess if the leukaemia data fits these distributions, i.e., DTEIW and EIW. The result of the $K-S$ test based on the $M L$ estimates shows that DTEIW and EIW distributions provide a plausible model for the data since the computed values (which are $K-S_{D T E I W}=0.1457$ and $K-S_{E I W}=0.2220$ ) are less than the theoretical value (which is $K-S_{0.05,20}=0.2940$ ), see Table 5 .

Then log-likelihood $(\ln L)$, root mean square error $(R M S E)$, coefficient of determination $\left(R^{2}\right)$ and Akaike information criterion $(A I C)$ are used to evaluate the goodness of fit of the DTEIW and EIW distributions to the data, and are calculated by the following formulas

and

$$
\begin{aligned}
& R M S E=\sqrt{\frac{1}{n} \sum_{i=1}^{n}\left(\hat{F}_{i}-u_{i}\right)^{2}}, \\
& R^{2}=1-\frac{\sum_{i=1}^{n}\left(\hat{F}_{i}-u_{i}\right)^{2}}{\sum_{i=1}^{n}\left(\hat{F}_{i}-\widehat{F}\right)^{2}}
\end{aligned}
$$

$$
A I C=-2 \ln L+2 p
$$

where $\hat{F}_{i}$ is the estimated value of the $c d f$ in (3) for $i$ th order statistic, $u_{i}$ is the expected value of the $\hat{F}_{i}$, $\overline{\hat{F}}$ is the mean of the $\widehat{F}_{i}$ as $\overline{\hat{F}}=\frac{1}{n} \sum_{i=1}^{n} \widehat{F}_{i}, \ln L$ is the maximized value of the log-likelihood function for the respective distribution and $p$ is the number of parameters to be estimated.

Table 5 presents the results of the $M L$ estimates of parameters $\alpha, \beta$ and $\theta$ and model evaluation tests which are $\ln L, R M S E, R^{2}$ and $A I C$ of DTEIW and EIW distributions for the given data. It shows that the $D T E I W$ distribution provides better fit to the data than the EIW distribution does, since lower values of $R M S E$ and $A I C$ and higher values of $\ln L$ and $R^{2}$ indicate better fitting to the data, see Table 5.

Table 5. Results of the $M L$ estimates of parameters and model evaluation tests for the data.

\begin{tabular}{ccccccccc}
\hline $\begin{array}{c}\text { Fitting } \\
\text { Distribution }\end{array}$ & $\hat{\alpha}_{M L}$ & $\hat{\beta}_{M L}$ & $\hat{\theta}_{M L}$ & $K-S$ & $\ln L$ & $R M S E$ & $R^{2}$ & \multirow{2}{*}{$A I C$} \\
\hline DTEIW & 1.9150 & 0.5905 & 1.6673 & 0.1457 & -22.9624 & 0.0671 & 0.9356 & 51.9248 \\
$E I W$ & 1.8112 & 2.0651 & 1.4377 & 0.2220 & -29.0867 & 0.1023 & 0.8365 & 64.1733 \\
\hline
\end{tabular}

\section{CONCLUSIONS}

In this study, a five-parameter DTEIW distribution with known truncation points is introduced. The exact formulas of the statistical properties, such as moments, characteristic function, moment generating function and Fisher information matrix are derived. We also obtain estimates of the 
parameters of DTEIW distribution using different estimation methods; $M L, L S$ and $W L S$. A MonteCarlo simulation study is used to compare the performances of the estimators with respect to different cases of parameters and different sample sizes.

Comparing the biases in the context of simulation results: (i) for parameter $\alpha$, it is observed that the $L S$ estimator performs best in almost all the cases considered for large sample size $n=100$; (ii) for parameter $\beta$, the $W L S$ and $M L$ estimators have best performance for $n=10$ and $n=20$, respectively; and (iii) for parameter $\theta$, the $M L$ estimator is the best among the others for moderate and large sample sizes.

For RMSE: (i) for parameter $\alpha$, the performance of the $M L$ estimator is best among the other estimation methods in almost all cases; (ii) for parameter $\beta$, the $M L$ estimator works best in all cases and all sample sizes $(n=10,20,50$ and 100); and (iii) for parameter $\theta$, when $n \geq 50$, the $M L$ estimator shows better performance when compared with the others in many cases.

Clearly, when $n \leq 20$, it can be seen that RMSE values of the $M L, L S$ and $W L S$ estimates are quite large for parameter $\theta$. Finally, for all methods, the bias and the $R M S E$ values decrease as sample size increases in all cases. Therefore, all the considered estimators of the parameters of DTEIW distribution are asymptotically unbiased and consistent.

Finally, the applicability of the DTEIW distribution is illustrated by an application to a real data set in which the DTEIW distribution has provided better fit than the EIW distribution.

\section{APPENDIX}

In this section, how certain integrals given in equations (17), (18) and (19) are obtained is explained.

A.1. The integral given in Equation (17) is get as follows

$$
\begin{aligned}
& E\left(\frac{\partial^{2} \ln L(\alpha, \beta, \theta)}{\partial \beta^{2}}\right)=-\frac{n}{\beta^{2}}+n \alpha \theta \frac{\left(t_{1}^{-\beta} \ln ^{2} t_{1} e^{-\alpha \theta t_{1}-\beta}\left(1-\alpha \theta t_{1}^{-\beta}\right)-t_{0}^{-\beta} \ln ^{2} t_{0} e^{-\alpha \theta t_{0}-\beta}\left(1-\alpha \theta t_{0}{ }^{-\beta}\right)\right)}{e^{-\alpha \theta t_{1}-\beta}-e^{-\alpha \theta t_{0}-\beta}} \\
& +n \alpha^{2} \theta^{2} \frac{\left(t_{1}-\beta \ln t_{1} e^{-\alpha \theta t_{1}-\beta}-t_{0}-\beta \ln t_{0} e^{-\alpha \theta t_{0}-\beta}\right)^{2}}{\left(e^{-\alpha \theta t_{1}-\beta}-e^{-\alpha \theta t_{0}-\beta}\right)^{2}} \\
& -\frac{n \alpha \theta}{e^{-\alpha \theta t_{1}-\beta}-e^{-\alpha \theta t_{0}}-\beta} \int_{t_{0}}^{t_{1}} x^{-\beta} \ln ^{2} x \alpha \theta \beta x^{-(\beta+1)} e^{-\alpha \theta x^{-\beta}} d x .
\end{aligned}
$$

If the transformation in (5) is applied to the integral $\int_{t_{0}}^{t_{1}} x^{-\beta}(\ln x)^{2} \alpha \theta \beta x^{-(\beta+1)} e^{-\alpha \theta x^{-\beta}} d x$, then we have

$$
\begin{aligned}
\int_{t_{0}}^{t_{1}} x^{-\beta} \ln ^{2} x \alpha \theta \beta x^{-(\beta+1)} e^{-\alpha \theta x^{-\beta} d x=} & \frac{\alpha \theta}{\beta^{2}} \int_{\alpha \theta t_{1}-\beta}^{\alpha \theta t_{0}-\beta} u^{-1}(\ln u-\ln (\alpha \theta))^{2} e^{-u} d u . \\
= & \frac{\alpha \theta}{\beta^{2}} \int_{\alpha \theta t_{0}-\beta}^{\alpha \theta t^{-\beta}} u^{-1}(\ln u-\ln (\alpha \theta))^{2}\left(\sum_{k=0}^{\infty} \frac{(-u)^{k}}{k !}\right) d u \\
= & \frac{\alpha \theta}{\beta^{2}} \sum_{k=0}^{\infty} \frac{(-1)^{k}}{k !} \int_{\alpha \theta t_{1}-\beta}^{\alpha \theta t_{0}-\beta} u^{k-1}(\ln u-\ln (\alpha \theta))^{2} d u \\
= & \frac{\alpha \theta}{\beta^{2}} \sum_{k=0}^{\infty} \frac{(-1)^{k}}{k !} \int_{\alpha \theta t_{0}-\beta}^{\alpha \theta t_{1}^{-\beta}} u^{k-1} \ln ^{2} u d u \\
& -\frac{2 \alpha \theta \ln \alpha \theta}{\beta^{2}} \sum_{k=0}^{\infty} \frac{(-1)^{k}}{k !} \int_{\alpha \theta t_{1}-\beta}^{\alpha \theta t_{0}-\beta} u^{k-1} \ln u d u \\
& +\frac{\alpha \theta \ln ^{2} \alpha \theta}{\beta^{2}} \sum_{k=0}^{\infty} \frac{(-1)^{k}}{k !} \int_{\alpha \theta t_{1}-\beta}^{\alpha \theta t_{1}-\beta} u^{k-1} d u .
\end{aligned}
$$


It should be noted that $\sum_{k=0}^{\infty} \frac{(-u)^{k}}{k !}$ is the maclaurin series of $e^{-u}$ given in (A2) and is used to compute a number of the following integrals. Here, $\int_{\alpha \theta t_{1}{ }^{-\beta}}^{\alpha \theta t^{-\beta}} u^{k-1} \ln u d u$ and $\int_{\alpha \theta t_{1}{ }^{-\beta}}^{\alpha \theta t^{-\beta}} u^{k-1} \ln ^{2} u d u$ in (A2) can be computed using equations (1.6.1.18) and (1.6.1.19) in Prudnikov et al. [22] as

$$
\int_{\alpha \theta t_{1}-\beta}^{\alpha \theta t_{0}-\beta} u^{k-1} \ln u d u=(\alpha \theta)^{k} t_{0}{ }^{-k \beta}\left(\frac{\ln \alpha \theta t_{0}-\beta}{n+1}-\frac{1}{(n+1)^{2}}\right)-(\alpha \theta)^{k} t_{1}{ }^{-k \beta}\left(\frac{\ln \alpha \theta t_{1}-\beta}{n+1}-\frac{1}{(n+1)^{2}}\right)
$$

and

$$
\begin{aligned}
\int_{\alpha \theta t_{1}-\beta}^{\alpha \theta t_{0}-\beta} u^{k-1} \ln ^{2} u d u= & (\alpha \theta)^{k} t_{0}-k \beta\left(\frac{\ln ^{2} \alpha \theta t_{0}-\beta}{n+1}-\frac{2 \ln \alpha \theta t_{0}-\beta}{(n+1)^{2}}+\frac{2}{(n+1)^{3}}\right) \\
& -(\alpha \theta)^{k} t_{1}-k \beta\left(\frac{\ln ^{2} \alpha \theta t_{1}-\beta}{n+1}-\frac{2 \ln \alpha \theta t_{1}-\beta}{(n+1)^{2}}+\frac{2}{(n+1)^{3}}\right),
\end{aligned}
$$

respectively. So, $E\left(\frac{\partial^{2} \ln L(\alpha, \beta, \theta)}{\partial \beta^{2}}\right)$ becomes

$$
\begin{aligned}
& E\left(\frac{\partial^{2} \ln L(\alpha, \beta, \theta)}{\partial \beta^{2}}\right)=-\frac{n}{\beta^{2}}+n \alpha \theta \frac{\left(t_{1}^{-\beta}\left(\operatorname{lnt} t_{1}\right)^{2} e^{-\alpha \theta t_{1}-\beta}\left(1-\alpha \theta t_{1}^{-\beta}\right)-t_{0}^{-\beta}\left(\operatorname{lnt} t_{0}\right)^{2} e^{-\alpha \theta t_{0}-\beta}\left(1-\alpha \theta t_{0}-\beta\right)\right)}{e^{-\alpha \theta t_{1}-\beta}-e^{-\alpha \theta t_{0}-\beta}} \\
& +n \alpha^{2} \theta^{2} \frac{\left(t_{1}^{-\beta} \operatorname{lnt}_{1} e^{-\alpha \theta t_{1}-\beta}-t_{0}-\beta \ln t_{0} e^{-\alpha \theta t_{0}-\beta}\right)^{2}}{\left(e^{-\alpha \theta t_{1}-\beta}-e^{-\alpha \theta t_{0}-\beta}\right)^{2}} \\
& -\frac{n \beta^{-2}}{e^{-\alpha \theta t_{1}-\beta}-e^{-\alpha \theta t_{0}}-\beta} \sum_{k=0}^{\infty} \frac{(-1)^{k}(\alpha \theta)^{k+2} t_{0}-k \beta}{k !}\left(\frac{\ln ^{2} \alpha \theta t_{0}-\beta}{n+1}-\frac{2 \ln \alpha \theta t_{0}-\beta}{(n+1)^{2}}+\frac{2}{(n+1)^{3}}\right) \\
& +\frac{n \beta^{-2}}{e^{-\alpha \theta t_{1}-\beta}-e^{-\alpha \theta t_{0}-\beta}} \sum_{k=0}^{\infty} \frac{(-1)^{k}(\alpha \theta)^{k+2} t_{1}-k \beta}{k !}\left(\frac{\ln ^{2} \alpha \theta t_{1}-\beta}{n+1}-\frac{2 \ln \alpha \theta t_{1}^{-\beta}}{(n+1)^{2}}+\frac{2}{(n+1)^{3}}\right) \\
& +\frac{2 n \beta^{-2} \ln \alpha \theta}{e^{-\alpha \theta t_{1}-\beta}-e^{-\alpha \theta t_{0}-\beta}} \sum_{k=0}^{\infty} \frac{(-1)^{k}(\alpha \theta)^{k+2} t_{0}{ }^{-k \beta}}{k !}\left(\frac{\ln \alpha \theta t_{0}-\beta}{n+1}-\frac{1}{(n+1)^{2}}\right) \\
& -\frac{2 n \beta^{-2} \ln \alpha \theta}{e^{-\alpha \theta t_{1}-\beta}-e^{-\alpha \theta t_{0}-\beta}} \sum_{k=0}^{\infty} \frac{(-1)^{k}(\alpha \theta)^{k+2} t_{1}-k \beta}{k !}\left(\frac{\ln \alpha \theta t_{1}-\beta}{n+1}-\frac{1}{(n+1)^{2}}\right) \\
& -\frac{n \beta^{-2} l^{2} \alpha \theta}{e^{-\alpha \theta t_{1}-\beta}-e^{-\alpha \theta t_{0}}{ }^{-\beta}} \sum_{k=0}^{\infty} \frac{(-1)^{k}(\alpha \theta)^{k+2}}{k ! k}\left(t_{0}{ }^{-k \beta}-t_{1}{ }^{-k \beta}\right) \text {. }
\end{aligned}
$$

A.2. The integral given in Equation (18) is obtained as follows

$$
\begin{aligned}
& E\left(\frac{\partial^{2} \ln L(\alpha, \beta, \theta)}{\partial \alpha \partial \beta}\right)=-n \theta \frac{\left(t_{1}^{-\beta} \ln t_{1} e^{-\alpha \theta t_{1}-\beta}\left(1-\alpha \theta t_{1}^{-\beta}\right)-t_{0}^{-\beta} \ln t_{0} e^{-\alpha \theta t_{0}-\beta}\left(1-\alpha \theta t_{0}{ }^{-\beta}\right)\right)}{e^{-\alpha \theta t_{1}-\beta}-e^{-\alpha \theta t_{0}-\beta}} \\
& -n \alpha \theta^{2} \frac{\left(t_{1}^{-\beta} \operatorname{lnt} t_{1} e^{-\alpha \theta t_{1}-\beta}-t_{0}{ }^{-\beta} \operatorname{lnt}_{0} e^{-\alpha \theta t_{0}-\beta}\right)\left(t_{1}{ }^{-\beta} e^{-\alpha \theta t_{1}-\beta}-t_{0}{ }^{-\beta} e^{-\alpha \theta t_{0}-\beta}\right)}{\left(e^{-\alpha \theta t_{1}-\beta}-e^{-\alpha \theta t_{0}-\beta}\right)^{2}} \\
& +\frac{n \theta}{e^{-\alpha \theta t_{1}-\beta}-e^{-\alpha \theta t_{0}-\beta}} \int_{t_{0}}^{t_{1}} x^{-\beta} \ln x \alpha \theta \beta x^{-(\beta+1)} e^{-\alpha \theta x^{-\beta}} d x,
\end{aligned}
$$

Here, $\int_{t_{0}}^{t_{1}} x^{-\beta} \ln x \alpha \theta \beta x^{-(\beta+1)} e^{-\alpha \theta x^{-\beta}} d x$ can be computed using the transformation in (5) as

$$
\begin{aligned}
\int_{t_{0}}^{t_{1}} x^{-\beta} \ln x \alpha \theta \beta x^{-(\beta+1)} e^{-\alpha \theta x^{-\beta}} d x= & \frac{\alpha \theta}{\beta^{2}} \int_{\alpha \theta t_{1}-\beta}^{\alpha \theta t_{0}-\beta} u^{-1}(\ln u-\ln (\alpha \theta)) e^{-u} d u \\
= & \frac{\alpha \theta}{\beta^{2}} \int_{\alpha \theta t_{1}-\beta}^{\alpha \theta t^{-\beta}} u^{-1}(\ln u-\ln (\alpha \theta))\left(\sum_{k=0}^{\infty} \frac{(-u)^{k}}{k !}\right) d u \\
= & \frac{\alpha \theta}{\beta^{2}} \sum_{k=0}^{\infty} \frac{(-1)^{k}}{k !} \int_{\alpha \theta t_{1}-\beta}^{\alpha \theta t_{0}-\beta} u^{k-1} \ln u d u \\
& -\frac{\ln (\alpha \theta) \alpha \theta}{\beta^{2}} \sum_{k=0}^{\infty} \frac{(-1)^{k}}{k !} \int_{\alpha \theta t_{1}-\beta}^{\alpha \theta t_{0}-\beta} u^{k-1} d u .
\end{aligned}
$$


Here, $\int_{\alpha \theta t_{1}{ }^{-\beta}}^{\alpha \theta t^{-\beta}} u^{k-1} \ln u d u$ given in (A3) can be computed using equation (1.6.1.18) in Prudnikov et al. [22]. Therefore, $E\left(\frac{\partial^{2} \ln L(\alpha, \beta, \theta)}{\partial \alpha \partial \beta}\right)$ becomes

$$
\begin{aligned}
& E\left(\frac{\partial^{2} \ln L(\alpha, \beta, \theta)}{\partial \alpha \partial \beta}\right)=-n \theta \frac{\left(t_{1}^{-\beta} \ln t_{1} e^{-\alpha \theta t_{1}-\beta}\left(1-\alpha \theta t_{1}^{-\beta}\right)-t_{0}^{-\beta} \operatorname{lnt}_{0} e^{-\alpha \theta t_{0}-\beta}\left(1-\alpha \theta t_{0}{ }^{-\beta}\right)\right)}{e^{-\alpha \theta t_{1}-\beta}-e^{-\alpha \theta t_{0}-\beta}} \\
& -n \alpha \theta^{2} \frac{\left(t_{1}^{-\beta} \operatorname{lnt} t_{1} e^{-\alpha \theta t_{1}-\beta}-t_{0}{ }^{-\beta} \operatorname{lnt}_{0} e^{-\alpha \theta t_{0}-\beta}\right)\left(t_{1}^{-\beta} e^{-\alpha \theta t_{1}-\beta}-t_{0}-\beta e^{-\alpha \theta t_{0}-\beta}\right)}{\left(e^{-\alpha \theta t_{1}-\beta}-e^{-\alpha \theta t_{0}}{ }^{-\beta}\right)^{2}} \\
& +\frac{n}{\beta^{2}\left(e^{-\alpha \theta t_{1}-\beta}-e^{-\alpha \theta t_{0}}{ }^{-\beta}\right)} \sum_{k=0}^{\infty} \frac{(-1)^{k} \alpha^{k+1} \theta^{k+2} t_{0}{ }^{-k \beta}}{k !}\left(\frac{\ln \alpha \theta t_{0}-\beta}{n+1}-\frac{1}{(n+1)^{2}}\right) \\
& -\frac{n}{\beta^{2}\left(e^{-\alpha \theta t_{1}-\beta}-e^{-\alpha \theta t_{0}}{ }^{-\beta}\right)} \sum_{k=0}^{\infty} \frac{(-1)^{k} \alpha^{k+1} \theta^{k+2} t_{1}{ }^{-k \beta}}{k !}\left(\frac{\ln \alpha \theta t_{1}-\beta}{n+1}-\frac{1}{(n+1)^{2}}\right) \\
& -\frac{n \ln \alpha \theta}{\beta^{2}\left(e^{-\alpha \theta t_{1}-\beta}-e^{-\alpha \theta t_{0}-\beta}\right)} \sum_{k=0}^{\infty} \frac{(-1)^{k} \alpha^{k+1} \theta^{k+2}}{k ! k}\left(t_{0}{ }^{-k \beta}-t_{1}{ }^{-k \beta}\right) \text {. }
\end{aligned}
$$

A.3. The integral given in Equation (19) is computed as follows

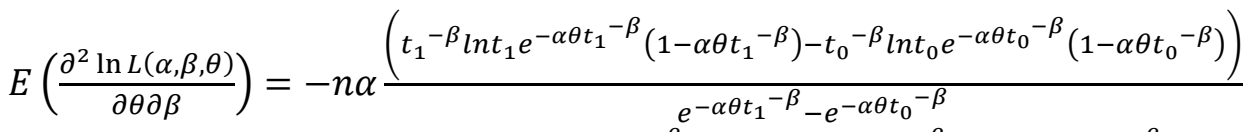

$$
\begin{aligned}
& -n \alpha^{2} \theta \frac{\left(t_{1}^{-\beta} \ln t_{1} e^{-\alpha \theta t_{1}-\beta}-t_{0}-\beta \ln t_{0} e^{-\alpha \theta t_{0}-\beta}\right)\left(t_{1}^{-\beta} e^{-\alpha \theta t_{1}-\beta}-t_{0}-\beta e^{-\alpha \theta t_{0}-\beta}\right)}{\left(e^{-\alpha \theta t_{1}-\beta}-e^{-\alpha \theta t_{0}-\beta}\right)^{2}} \\
& +\frac{n \alpha}{e^{-\alpha \theta t_{1}-\beta}-e^{-\alpha \theta t_{0}}-\beta} \int_{t_{0}}^{t_{1}} x^{-\beta} \ln x \alpha \theta \beta x^{-(\beta+1)} e^{-\alpha \theta x^{-\beta}} d x .
\end{aligned}
$$

Using the transformation in (5) and equation (1.6.1.18) in Prudnikov et al. [22], we obtain the following

$$
\begin{aligned}
& E\left(\frac{\partial^{2} \ln L(\alpha, \beta, \theta)}{\partial \theta \partial \beta}\right)=-n \alpha \frac{\left(t_{1}^{-\beta} \ln t_{1} e^{-\alpha \theta t_{1}^{-\beta}}\left(1-\alpha \theta t_{1}^{-\beta}\right)-t_{0}^{-\beta} \ln t_{0} e^{-\alpha \theta t_{0}-\beta}\left(1-\alpha \theta t_{0}^{-\beta}\right)\right)}{e^{-\alpha \theta t_{1}^{-\beta}}-e^{-\alpha \theta t_{0}-\beta}} \\
& -n \alpha^{2} \theta \frac{\left(t_{1}^{-\beta} \ln t_{1} e^{-\alpha \theta t_{1}-\beta}-t_{0}{ }^{-\beta} \operatorname{lnt}_{0} e^{-\alpha \theta t_{0}-\beta}\right)\left(t_{1}^{-\beta} e^{-\alpha \theta t_{1}-\beta}-t_{0}-\beta e^{-\alpha \theta t_{0}-\beta}\right)}{\left(e^{-\alpha \theta t_{1}-\beta}-e^{-\alpha \theta t_{0}-\beta}\right)^{2}} \\
& +\frac{n}{\beta^{2}\left(e^{-\alpha \theta t_{1}-\beta}-e^{-\alpha \theta t_{0}}-\beta\right)} \sum_{k=0}^{\infty} \frac{(-1)^{k} \alpha^{k+2} \theta^{k+1} t_{0}{ }^{-k \beta}}{k !}\left(\frac{\ln \alpha \theta t_{0}-\beta}{n+1}-\frac{1}{(n+1)^{2}}\right) \\
& -\frac{n}{\beta^{2}\left(e^{-\alpha \theta t_{1}-\beta}-e^{-\alpha \theta t_{0}}-\beta\right.} \sum_{k=0}^{\infty} \frac{(-1)^{k} \alpha^{k+2} \theta^{k+1} t_{1}-k \beta}{k !}\left(\frac{\ln \alpha \theta t_{1}-\beta}{n+1}-\frac{1}{(n+1)^{2}}\right) \\
& -\frac{n \ln \alpha \theta}{\beta^{2}\left(e^{-\alpha \theta t_{1}-\beta}-e^{-\alpha \theta t_{0}}{ }^{-\beta}\right)} \sum_{k=0}^{\infty} \frac{(-1)^{k} \alpha^{k+2} \theta^{k+1}}{k ! k}\left(t_{0}{ }^{-k \beta}-t_{1}{ }^{-k \beta}\right) \text {. }
\end{aligned}
$$

\section{REFERENCES}

[1] Afify AZ, Nofal ZM, Butt NS. Transmuted complementary Weibull geometric distribution. Pakistan Journal of Statistics and Operation Research 2014; 10(4): 435-454.

[2] Ahmad AA. Moments of order statistics from doubly truncated continuous distribution. Statistics 2001; 35: 479-494. 
[3] Ali MM, Pal M, Woo JS. Some exponentiated distributions. Communications for Statistical Applications and Methods 2007; 14(1): 93-109.

[4] Al-Matrafi BN, Jawa TM. Recurrence relations for single moments of generalized order statistics from doubly truncated continuous distributions. Journal of King Abdulaziz University: Science 2008; 20(2): 79-89.

[5] Calabria R, Pulcini G. Confidence limits for reliability and tolerance limits in the inverse Weibull distribution. Reliability Engineering \& System Safety 1989; 24(1): 77-85.

[6] Cohen AC, Whitten BJ. Parameter estimation in reliability and life span models. New York and Basel: Marcel Dekker Inc., 1988.

[7] Coffey CS, Muller KE. Properties of doubly-truncated gamma variables. Communications in Statistics-Theory and Methods 2000; 29(4): 851-857.

[8] de Gusmão FR, Ortega EM, Cordeiro, GM. The generalized inverse Weibull distribution. Statistical Papers 2011; 52(3): 591-619.

[9] Foss S, Korshunov D, Zachary S. An introduction to heavy-tailed and subexponential distributions. New York: Springer, 2011.

[10] Gupta RC, Gupta PL, Gupta RD. Modeling failure time data by Lehman alternatives. Communications in Statistics, Theory and Methods 1998; 27: 887-904.

[11] Gupta RD, Kundu D. Exponentiated exponential family: an alternative to gamma and Weibull distributions. Biometrical Journal 2001; 43: 117-130.

[12] Jiang R, Murthy DNP, Ji P. Models involving two inverse Weibull distributions. Reliability Engineering \& System Safety 2001; 73(1): 73-81.

[13] Johnson NL, Kotz S, Balakrishnan N. Continuous Univariate Distribution, Vol. 2, 2nd edition, New York: Wiley, 1995.

[14] Joshi PC. A note on the moments of order statistics from doubly truncated exponential distribution. Annals of the Institute of Statistical Mathematics 1979; 31: 321-324.

[15] Keller AZ, Kamath ARR, Perera UD. Reliability analysis of CNC machine tools. Reliability Engineering 1982; 3: 449-473.

[16] Kantar YM, Usta I. Analysis of the upper-truncated Weibull distribution for wind speed. Energy Conversion and Management 2015; 96: 81-88.

[17] Khan MS, Pasha GR, Pasha AH. Theoretical analysis of inverse Weibull distribution.WSEAS Transactions on Mathematics 2008; 7(2): 30-38.

[18] Lawless JF. Statistical models and methods for lifetime data. New York: Wiley, 1982.

[19] Marusic M, Markovic D, Jukic D. Least squares fitting the three-parameter inverse Weibull density. Mathematical Communications 2010; 15(2): 539-553. 
[20] Nadarajah S, Kotz S. The exponentiated type distributions. Acta Applicandae Mathematicae 2006; 92(2): 97-111.

[21] Okasha MK, Alqanoo IM. Inference on the doubly truncated Gamma distribution for lifetime data. International Journal of Mathematics and Statistics Invention 2014; 2: 1-17.

[22] Prudnikov AP, Brychkov YA, Marichev, OI. Integrals and series, vol 1. Amsterdam: Gordon and Breach Science Publishers, 1986.

[23] Shah SM, Jaiswal MC. Estimation of parameters of doubly truncated normal distribution from first four sample moments. Annals of the Institute of Statistical Mathematics 1966; 18(1): 107111.

[24] Swain J, Venkatraman S, Wilson J. Least-squares estimation of distribution function in Johnson's translation system. Journal of Statistical Computation and Simulation 1988; 29: 271-297.

[25] Zhang T, Xie M. On the upper truncated Weibull distribution and its reliability implications. Reliability Engineering \& System Safety 2011; 96(1): 194-200. 